\title{
Fusing the Seth-Hill strain tensors to fit compressible elastic material responses in the nonlinear regime
}

\author{
L.A.A. Beex \\ Institute of Computational Engineering, Faculty of Science, Technology and \\ Communication, University of Luxembourg, Maison du Nombre, 6 Avenue de la Fonte, \\ L-4364, Esch-sur-Alzette, Luxembourg.
}

\begin{abstract}
Strain energy densities based on the Seth-Hill strain tensors are often used to describe the hyperelastic mechanical behaviours of isotropic, transversely isotropic and orthotropic materials for relatively large deformations. Since one parameter distinguishes which strain tensor of the Seth-Hill family is used, one has in theory the possibility to fit the material response in the nonlinear regime. Most often for compressible deformations however, this parameter is selected such that the Hencky strain tensor is recovered, because it yields rather physical stress-strain responses. Hence, the response in the nonlinear regime is in practise not often tailored to match experimental data. To ensure that elastic responses in the nonlinear regime can more accurately be controlled, this contribution proposes three generalisations that combine several Seth-Hill strain tensors. The generalisations are formulated such that the stress-strain responses for infinitesimal deformations remain unchanged. Consequently, the identification of the Young's moduli, Poisson's ratios and shear moduli is not affected. 3D finite element simulations are performed for isotropy and orthotropy, with an emphasis on the identification of the new material parameters.
\end{abstract}

Key words: hyperelasticity, Seth-Hill strain tensors, Hencky strain tensor, parameter identification, isotropy, orthotropy, data driven modelling

*Email: lars.beex@uni.lu, L.A.A.Beex@gmail.com 


\section{Introduction}

Elasticity can be subdivided into hyperelasticity and hypoelasticity. Hyperelasticity is true elasticity, which entails that all energy supplied to the material is stored in the material. In case of hypoelasticity on the other hand, energy may undesirably be dissipated. In order to investigate if a stress-strain relation can exactly be integrated into a strain energy density and hence, to investigate if a constitutive model is hyperelastic, Riemannian geometry was used by Bernstein (1960a,b).

Hyperelasticity is still often associated with incompressible materials (Treloar, 1958; Ogden, 1972), because it was originally proposed to treat the incompressibility condition as a constraint in constrained minimization (Zienkiewicz \& Taylor, 2000). These days compressible hyperelasticity is however also frequently used. The increase of man-made materials that can undergo large elastic compressible deformations (Wismans et al., 2010; Schraedler et al., 2011; Kucheyev et al., 2012; Pokorný et al., 2017), as well as the increased interest in biological tissues that can undergo large elastic compressible deformations (Cotin et al., 1999; Baaijens et al., 2005; Hrapko et al., 2006; Carniel \& Fancello, 2017), is partially responsible for this. In contrast to hypoelasticity (Truesdell, 1955; Khan et al., 2010; Beex \& Peerlings, 2012), hyperelasticity also allows the formulation of error estimators in terms of stored and dissipated energies (Lovadina \& Stenberg, 2006; Bui et al., 2018), which are invariant scalars, and it avoids erroneous energy dissipation of dissipative material models (Håkanson et al., 2005; Harrysson \& Ristinmaa, 2008; Loew et al., 2019). Hyperelasticity is thus not only important for incompressible materials, but also for compressible materials. It is also not only important for purely elastic constitutive descriptions, but also for dissipative constitutive descriptions.

A frequently employed compressible hyperelastic model uses a fourthorder stiffness tensor and one of the strain tensors of the Seth-Hill family in the strain energy density function, because it allows to describe the mechanical responses of isotropic, transversely isotropic and orthotropic materials. One parameter ( $a$, see Fig. 1) distinguishes which of the Seth-Hill strain tensors is used. This choice affects only the nonlinear regime, because in the infinitesimal limit any of the Seth-Hill strain tensors recover the same response.

In theory, one thus has the opportunity to tailor material parameter $a$ to fit the constitutive description to experimentally observed stress-deformation 
curves. In practise however, one either uses the Green-Lagrangian strain tensor $(a=2)$ because of its ease of implementation, or the Hencky strain tensor (i.e. the logarithmic strain tensor, natural strain tensor, true strain tensor) (Miehe et al., 2002; Andrade et al., 2011; Mahnken \& Shaban, 2013; Vogel et al., 2014) because associated stresses are monotonically increasing (see Fig. 1). Stresses associated with $a>0$ have the problem that they do not approach $-\infty$ when the deformation tends to large compressions. In those cases, finite element simulations will fail to converge due to the lack of stiffness. Stresses $a<0$ have the same problem, but in tension (see Fig. 1). In practise, the Hencky strain tensor is thus often used and a true parameter identification for the nonlinear regime does not take place. Note that the Lagrangian Hencky strain tensor is considered here (Miehe \& Lambrecht, 2001), not its Eulerian counterpart (Geers, 2004; Javani et al., 2014) for which, if used appropriately, it has been shown to meet Bernstein's integrability condition (Xiao et al., 1997; Xiao and Chen, 2002).
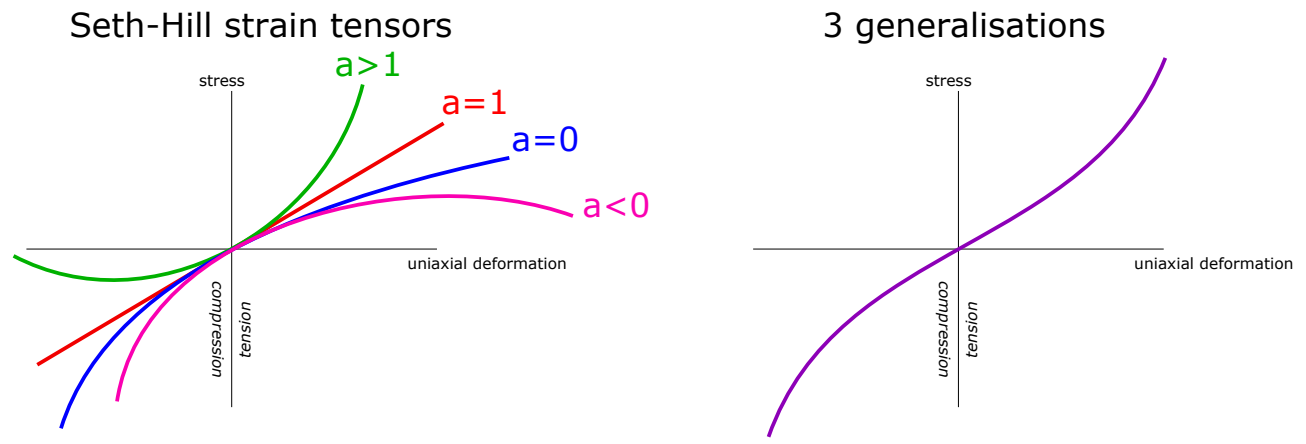

Figure 1: Sketch of stress-uniaxial deformation curves possible with the Seth-Hill strain tensors (left) and one that is not possible with the Seth-Hill strain tensors (right), but it is possible with the proposed generalisations.

To make sure that responses as presented on the right in Fig. 1 can be captured and can truly be fitted, one may consider the material parameters to be dependent on the deformation. This has the advantage that the Poynting effect can accurately be identified and incorporated (see e.g. Mihai \& Goriely (2011, 2017) and De Rosa et al. (2017)).

This contribution argues however that instead of developing new models, a solution to achieve a good correspondence between the experimental data and the model response may be sought in the combination of several 
hyperelastic models. In this way, one shifts the complexity from formulating new material models to employing and/or developing numerical strategies required to identify material parameters. This ansatz would fit the current interest in 'Data Driven Modelling'.

Recently, Korobeynikov (2018) has proposed such a generalised combination for hyperelasticity by collecting several strain tensors of different families in a single strain tensor. In contrast to Korobeynikov (2018), the aim of the current contribution is to only fuse the strain tensors of the Seth-Hill family (Doyle \& Ericksen, 1956; Seth, 1962; Hill, 1968, 1978; Curnier \& Rakotomanana, 1991; Miehe \& Lambrecht, 2001; Schröder et al., 2002; Latorre \& Montáns, 2016). The advantages of limiting one to the Seth-Hill family are that not only isotropic, but also transversely isotropic and orthotropic material responses can be described, and that one can employ the efficient implementation for the stresses and tangent stiffness tensors offered by Miehe \& Lambrecht (2001).

Three generalisations are proposed in the current contribution. First, the strain energy density can be constructed as a weighted sum of conventional strain energy densities, where 'conventional' refers to the choice of a single strain tensor of the Seth-Hill family. Second, the strain tensor itself can be constructed as a weighted sum of conventional strain tensors. Third, the weights for the construction of a new strain tensor based on several conventional strain tensors can be made dependent on the volume change (although probably any, or any combination, of the invariants of the deformation would work). By ensuring that the sum of the weights equals one for the generalisations, the infinitesimal theory is recovered, which is not the case for many anisotropic hyperelastic models (see e.g. De Rosa et al. (2017) for a discussion on this). In other words, the identification of the conventional material parameters in the fourth-order stiffness tensor (i.e. the Young's moduli, Poisson's ratios and possibly the shear moduli) remains unchanged. Additional identification efforts are however required to identify the weights (amongst others).

The generalisations' common denominator with Ogden's models (Ogden, $1972,1984)$ is that the strain energy densities are expressed in terms of a sum of the exponentations of the principle stretches. The difference is that the generalisations' strain energy densities use stiffness tensors, ensuring that the generalisations can treat isotropy, transverse isotropy and orthotropy.

In the light of the trend of 'Data Driven Modelling', it may also be of interest to compare the proposed generalisations with What-You-Prescribe- 
Is-What-You-Get (WYPiWYG) hyperelasticity (Crespo et al., 2016; Latorre \& Montáns, 2017; De Rosa et al., 2017; Romero et al., 2017). WYPiWYG hyperelasticity is a data-driven approach, in which measurement pairs of deformations and stresses are directly incorporated and a true fitting of material parameters is not required. Generally it seems that advantages of WYPiWYG hyperelasticity compared to the proposed generalisations (or any non-data-driven approach for that matter) come with disadvantages and vice versa.

For instance, WYPiWYG hyperelasticity automatically incorporates tensioncompression asymmetry, whereas only the third generalisation is able to do this to some extent (based on the volume change). WYPiWYG hyperelasticity also automatically incorporates dependencies between shear and nonshear parts of the deformations, whereas none of the proposed generalisations are able to do so. At the same time, because measurement pairs are directly incorporated in WYPiWYG hyperelasticity, measurement noise has an impact on the computational efficiency of the FE simulations. Filtering can then be used, which is currently under investigation (Latorre \& Montáns, 2018). Second, WYPiWYG hyperelasticity will not incorporate unconventional dependencies in the strain energy density (which the proposed generalisations are not able to), if the dependencies are not sampled in the experiments. An open question for WYPiWYG hyperelasticity therefore seems to be which experiments are required and if they can be performed.

The outline of this paper is as follows. The conventional hyperelastic models based on the Seth-Hill family of strain tensors are described in section 2 . In section 3, the three generalisations are proposed. Section 4 discusses the finite element implementation for quasi-static finite element problems, excluding body forces. Section 5 presents several numerical results and focuses amongst others on the identification of the new material parameters. Section 6 ends this contribution with conclusions. First, the notation is briefly summarised.

\section{Notation}

- scalars are denoted by small case and capital letters (e.g. $\alpha, a, A$ ),

- sets are denoted by caligraphic capital letters (e.g. $\left.\mathcal{A}=\left\{a_{1}, a_{2}, \ldots, a_{n}\right\}\right)$,

- columns are denoted by bars under letters (e.g. $\underline{a}, \underline{A})$,

- matrices are denoted by two bars under letters (e.g. $\underline{\underline{A}}$ ), 
- transposes of columns and matrices are denoted by superscript $T$ (e.g. $\underline{a}^{T}$, $\left.\underline{\underline{A}}^{T}\right)$,

- the $i^{\text {th }}$ base vector is denoted $\vec{e}_{i}$,

- a vector is denoted by an arrow on top of a letter (e.g. $\vec{a}=a_{i} \vec{e}_{i}$ ),

- Einstein's summation convention is used but it is avoided in case of possible misconceptions,

- a dyadic product is denoted by directly placing two vectors or tensors behind each other (i.e. $\vec{a} \vec{b}=a_{i} b_{j} \vec{e}_{i} \vec{e}_{j}$ ),

- $2^{\text {nd }}$-order tensors are denoted by bold capitals and bold Greek letters (e.g. $\mathbf{A}=A_{i j} \vec{e}_{i} \vec{e}_{j}$ and $\boldsymbol{\epsilon}=\epsilon_{i j} \vec{e}_{i} \vec{e}_{j}$ ),

- I denotes the $2^{\text {nd }}$-order unit tensor $\left(\mathbf{I}=\delta_{i j} \vec{e}_{i} \vec{e}_{j}\right.$, where $\delta_{i j}$ denotes the Kronecker delta),

- $n^{\text {th }}$-order tensors with $n>2$ are denoted by bold capitals with $n$ as a a left superscript (e.g. ${ }^{4} \mathbf{A}=A_{i j k l} \vec{e}_{i} \vec{e}_{j} \vec{e}_{k} \vec{e}_{l}$ ),

- right superscript $c$ at a $2^{\text {nd }}$-order tensor denotes its conjugate transpose (e.g. $\left.\mathbf{A}^{c}=A_{i j} \vec{e}_{j} \vec{e}_{i}\right)$,

- right superscripts between square brackets denote remaining conjugate transposes, where commas are used to denote the sequence (e.g. ${ }^{4} \mathbf{A}^{[13]}=$ $\left.A_{i j k l} \vec{e}_{k} \vec{e}_{j} \vec{e}_{i} \vec{e}_{l},{ }^{4} \mathbf{A}^{[13,14]}=A_{i j k l} \vec{e}_{l} \vec{e}_{j} \vec{e}_{i} \vec{e}_{k}, \mathbf{A}^{c}=\mathbf{A}^{[12]}\right)$,

- a dot between vectors and/or tensors denotes their inner product (e.g. A. $\left.\mathbf{B}=A_{i k} B_{k j} \vec{e}_{i} \vec{e}_{j}\right)$,

- a double dot between tensors denotes their double inner product (e.g. A : $\left.\mathbf{B}=A_{i j} B_{j i}\right)$,

- right superscript -1 at a $2^{\text {nd }}$-order tensor denotes its inverse (e.g. $\mathbf{A}^{-1}$. $\left.\mathbf{A}=\mathbf{A} \cdot \mathbf{A}^{-1}=\mathbf{I}\right)$,

- $\vec{X}$ denotes the vector pointing to a material point in the reference configuration $\left(\vec{X}=X_{i} \vec{e}_{i}\right)$,

- $\vec{u}$ denotes the displacement vector between a material point in the reference configuration and in a deformed configuration $\left(\vec{u}=u_{i} \vec{e}_{i}\right)$,

- $\vec{\nabla}_{0}$ denotes the vector differential operator with respect to the reference configuration $\left(\vec{\nabla}_{0}=\vec{e}_{i} \frac{\partial}{\partial X_{i}}\right)$, and

- $\mathbf{F}$ denotes the deformation gradient tensor $\left(\mathbf{F}=\mathbf{I}+\left(\vec{\nabla}_{0} \vec{u}\right)^{c}\right)$.

\section{Hyperelasticity based on the Seth-Hill family of strain tensors}

This section briefly discusses hyperelastic models based on the Seth-Hill family of (Lagrangian) strain tensors, also known as the Doyle-Ericksen family, which includes the Hencky strain tensor, the Biot strain tensor and the 
Green-Lagrangian strain tensor. As such, this section effectively summarises a substantial part of Miehe \& Lambrecht (2001) (and references therein) and is merely included to present a self-contained contribution.

The set of hyperelastic material models of interest here is given by the following strain energy density:

$$
W_{a}(\mathbf{F})=\frac{1}{2} \boldsymbol{\epsilon}_{a}:{ }^{4} \mathbf{Z}: \boldsymbol{\epsilon}_{a},
$$

where ${ }^{4} \mathbf{Z}$ denotes the well known $4^{\text {th }}$-order stiffness tensor with 21 nonzero components, containing two independent parameters in case of isotropy and six independent parameters in case of orthotropy. It is furthermore characterised by minor symmetries ${ }^{4} \mathbf{Z}={ }^{4} \mathbf{Z}^{[12]},{ }^{4} \mathbf{Z}={ }^{4} \mathbf{Z}^{[34]}$ (hence, ${ }^{4} \mathbf{Z}=$ $\left.{ }^{4} \mathbf{Z}^{[13,24]}\right)$.

$\boldsymbol{\epsilon}_{a}$ furthermore denotes one of the invariant strain tensors of the Seth-Hill family according to:

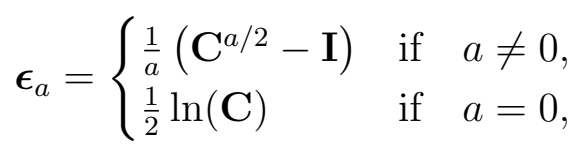

where $a$ denotes a real number $(a \in \mathbb{R})$ and $\mathbf{C}=\mathbf{F}^{c} \cdot \mathbf{F}$ denotes Green's deformation tensor. The Hencky strain tensor is recovered for $a=0$, the Biot strain tensor for $a=1$ (i.e. the linear strain tensor) and the GreenLagrangian strain tensor for $a=2$.

To determine these strain tensors, the three eigenvalues of Green's deformation tensor, $c_{i}$, and the associated eigenvectors, $\vec{n}_{i}$, are computed such that Green's deformation tensor can be written as:

$$
\mathbf{C}=\sum_{i=1}^{3} c_{i} \vec{n}_{i} \vec{n}_{i}
$$

The strain tensors of the Seth-Hill family can then be written as:

$$
\boldsymbol{\epsilon}_{a}=\sum_{i=1}^{3} \epsilon_{i} \vec{n}_{i} \vec{n}_{i}
$$

with

$$
\epsilon_{i}=\left\{\begin{array}{lll}
\frac{1}{a}\left(c_{i}^{a / 2}-1\right) & \text { if } & a \neq 0, \\
\frac{1}{2} \ln \left(c_{i}\right) & \text { if } & a=0 .
\end{array}\right.
$$




\subsection{Stress tensors}

The $1^{\text {st }}$ Piola-Kirchhoff stress tensor can be computed by differentiating the strain energy densities of Eq. (1) with respect to the deformation gradient tensor. After accounting for some (minor) symmetries and applying some tensor algebra, it can be written as follows:

$$
\mathbf{P}_{a}=\frac{\partial W_{a}}{\partial \mathbf{F}}=2 \mathbf{F} \cdot\left({ }^{4} \mathbf{Z}: \boldsymbol{\epsilon}_{a}: \frac{\partial \boldsymbol{\epsilon}_{a}}{\partial \mathbf{C}}\right)
$$

with

$$
\frac{\partial \boldsymbol{\epsilon}_{a}}{\partial \mathbf{C}}=\sum_{i=1}^{3} \frac{d_{i}}{2} \vec{n}_{i} \vec{n}_{i} \vec{n}_{i} \vec{n}_{i}+\sum_{i=1}^{3} \sum_{j \neq i}^{3} \frac{\theta_{i j}}{2}\left(\vec{n}_{i} \vec{n}_{j} \vec{n}_{i} \vec{n}_{j}+\vec{n}_{i} \vec{n}_{j} \vec{n}_{j} \vec{n}_{i}\right)
$$

where:

$$
\begin{aligned}
& d_{i}=c_{i}^{(a / 2)-1},
\end{aligned}
$$

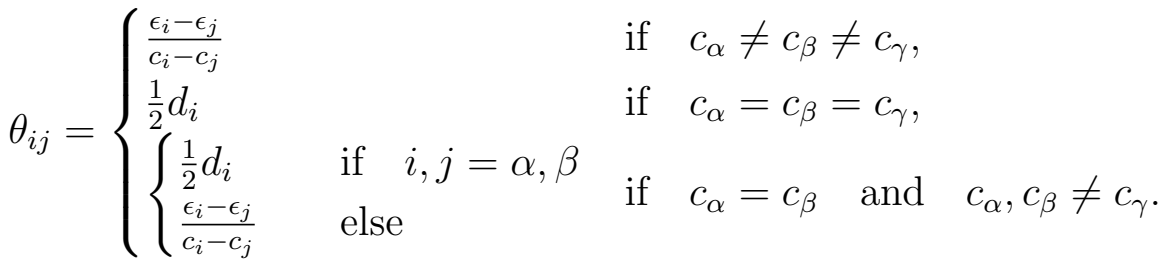

It is worth noting that $4^{\text {th }}$-order tensor $\frac{\partial \boldsymbol{\epsilon}_{a}}{\partial \mathbf{C}}$ only contains 45 independent components due to minor symmetry $\frac{\partial \boldsymbol{\epsilon}_{a}}{\partial \mathbf{C}}=\left(\frac{\partial \boldsymbol{\epsilon}_{a}}{\partial \mathbf{C}}\right)^{[34]}$.

Cauchy stress tensor $\boldsymbol{\sigma}_{a}$ can be computed based on $1^{\text {st }}$ Piola-Kirchhoff stress tensor $\mathbf{P}_{a}$ as follows:

$$
\boldsymbol{\sigma}_{a}=\frac{1}{J} \mathbf{P}_{a} \cdot \mathbf{F}^{c}
$$

where $J=\operatorname{det}(\mathbf{F})>0$ denotes the volume ratio.

It it worth mentioning that all possible Cauchy stress tensors that yield from this class of strain energy densities (i.e. regardless of the choice of $a$ ) abide the balance of linear momentum (i.e. the Cauchy stress tensors are symmetric), are all frame-indifferent (i.e. objective) and in the infinitesimal limit all behave in the same way (because all strain tensors of the Seth-Hill family tend to the infinitesimal strain tensor for $\mathbf{F} \approx \mathbf{I}$ ) . 


\subsection{Tangent stiffness tensor}

As the tangent stiffness tensor for any choice of $a$ is needed in a finite element framework, it is presented here. After employing some tensor algebra and accounting for (minor) symmetries, tangent stiffness tensor ${ }^{4} \mathbf{K}_{a}$ can be written as:

$$
\begin{aligned}
& { }^{4} \mathbf{K}_{a}=\frac{\partial^{2} W_{a}}{\partial(\mathbf{F})^{2}}=\frac{\partial \mathbf{P}_{a}}{\partial \mathbf{F}}=2\left({ }^{4} \mathbf{Z}: \boldsymbol{\epsilon}_{a}: \frac{\partial \boldsymbol{\epsilon}_{a}}{\partial \mathbf{C}} \mathbf{I}\right)^{[14]}+ \\
& 4 \mathbf{F} \cdot\left({ }^{4} \mathbf{Z}: \boldsymbol{\epsilon}_{a}: \frac{\partial^{2} \boldsymbol{\epsilon}_{a}}{\partial(\mathbf{C})^{2}} \cdot \mathbf{F}^{c}\right)^{[34]}+4 \mathbf{F} \cdot\left(\frac{\partial \boldsymbol{\epsilon}_{a}}{\partial \mathbf{C}}\right)^{[13,24]}:\left({ }^{4} \mathbf{Z}: \frac{\partial \boldsymbol{\epsilon}_{a}}{\partial \mathbf{C}} \cdot \mathbf{F}^{c}\right)^{[34]}
\end{aligned}
$$

where:

$$
\begin{array}{r}
\frac{\partial^{2} \boldsymbol{\epsilon}_{a}}{\partial(\mathbf{C})^{2}}=\sum_{i=1}^{3} \frac{g_{i}}{4} \vec{n}_{i} \vec{n}_{i} \vec{n}_{i} \vec{n}_{i} \vec{n}_{i} \vec{n}_{i}+\sum_{i=1}^{3} \sum_{j \neq i}^{3} \frac{\xi_{i j}}{4}\left({ }^{6} \mathbf{H}_{i j j}+{ }^{6} \mathbf{H}_{j i j}+{ }^{6} \mathbf{H}_{j j i}\right)+ \\
\sum_{i=1}^{3} \sum_{j \neq i}^{3} \sum_{k \neq i, k \neq j}^{3} \frac{\eta}{4}{ }^{6} \mathbf{H}_{i j k},
\end{array}
$$

with:

$$
\begin{aligned}
& g_{i}=(a-2) c_{i}^{(a / 2)-2},
\end{aligned}
$$

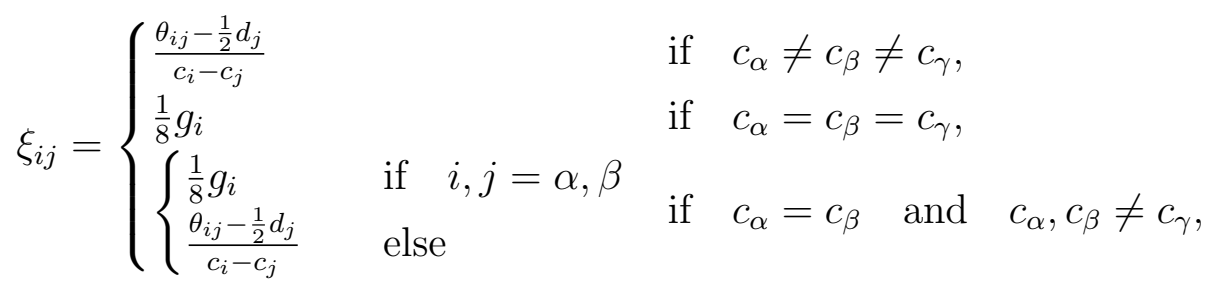

$$
\begin{aligned}
& \eta= \begin{cases}\frac{\epsilon_{1}}{\left(c_{1}-c_{2}\right)\left(c_{1}-c_{3}\right)}+\frac{\epsilon_{2}}{\left(c_{2}-c_{1}\right)\left(c_{2}-c_{3}\right)}+\frac{\epsilon_{3}}{\left(c_{3}-c_{1}\right)\left(c_{3}-c_{2}\right)} & \text { if } c_{\alpha} \neq c_{\beta} \neq c_{\gamma}, \\
\frac{1}{8} g_{i} & \text { if } c_{\alpha}=c_{\beta}=c_{\gamma}, \\
\xi_{\gamma \alpha} & \text { if } c_{\alpha}=c_{\beta} \text { and } c_{\alpha}, c_{\beta} \neq c_{\gamma},\end{cases}
\end{aligned}
$$




$$
\begin{aligned}
{ }^{6} \mathbf{H}_{i j k} & =\vec{n}_{i} \vec{n}_{j} \vec{n}_{i} \vec{n}_{k} \vec{n}_{j} \vec{n}_{k}+\vec{n}_{i} \vec{n}_{j} \vec{n}_{i} \vec{n}_{k} \vec{n}_{k} \vec{n}_{j}+\vec{n}_{i} \vec{n}_{j} \vec{n}_{k} \vec{n}_{i} \vec{n}_{j} \vec{n}_{k}+\vec{n}_{i} \vec{n}_{j} \vec{n}_{k} \vec{n}_{i} \vec{n}_{k} \vec{n}_{j}+ \\
& \vec{n}_{j} \vec{n}_{i} \vec{n}_{i} \vec{n}_{k} \vec{n}_{j} \vec{n}_{k}+\vec{n}_{j} \vec{n}_{i} \vec{n}_{i} \vec{n}_{k} \vec{n}_{k} \vec{n}_{j}+\vec{n}_{j} \vec{n}_{i} \vec{n}_{k} \vec{n}_{i} \vec{n}_{j} \vec{n}_{k}+\vec{n}_{j} \vec{n}_{i} \vec{n}_{k} \vec{n}_{i} \vec{n}_{k} \vec{n}_{j}
\end{aligned}
$$

Note that $6^{\text {th }}$-order tensor $\frac{\partial^{2} \boldsymbol{\epsilon}_{a}}{\partial(\mathbf{C})^{2}}$ only contains 56 independent components (of a total of 729 components), thanks to several minor symmetries.

\section{Generalisations}

As mentioned before, $a$ is often selected to be zero such that the Hencky strain tensor is recovered thanks to its physical properties and robustness in $\mathrm{FE}$ computations. In that case, the nonlinear responses are rigidly determined and cannot be altered to match experimental data. In this section, the three generalisations of the aforementioned hyperelastic models are proposed that provide more freedom to alter the material behaviour in the nonlinear regime.

\subsection{Fused strain energy densities}

In the first generalisation, the total strain energy density is considered to be a weighted sum of strain energy densities associated with the Seth-Hill family of strain tensors. A new strain energy density, $W$, is constructed that incorporates several strain energy densities of Eq. (1), $W_{a}$, as follows:

$$
W=\sum_{a \in \mathcal{A}} r_{a} W_{a}
$$

with

$$
\begin{aligned}
& \mathcal{A}=\left\{a_{1}, a_{2}, \ldots, a_{n}\right\}, \\
& \sum_{a \in \mathcal{A}} r_{a}=1,
\end{aligned}
$$

where $W_{a}$ denotes the strain energy density for a particular choice of $a \in \mathbb{R}$ of the conventional hyperelastic models of the previous section (see Eq. (1)). Its associated weight factor is denoted $r_{a}$. By ensuring that the sum of the weight factors equals one, the mechanical responses for infinitesimally small deformations remains the same as for the conventional hyperelasticity (for 
any choice of $a$ ). If set $\mathcal{A}$ furthermore contains a single value, the generalisation reduces to the conventional hyperelasticity of the previous section.

The $1^{\text {st }}$ Piola-Kirchhoff stress tensor that results from this generalisation can straightforwardly be computed based on the previous section as:

$$
\mathbf{P}=\sum_{a \in \mathcal{A}} r_{a} \mathbf{P}_{a}
$$

and its tangent stiffness tensor as:

$$
{ }^{4} \mathbf{K}=\sum_{a \in \mathcal{A}} r_{a}{ }^{4} \mathbf{K}_{a}
$$

\subsection{Fused strain tensors}

The second generalisation proposes to not consider the strain energy density as a weighted sum, but the strain tensor instead. We thus write the strain energy density as:

$$
W=\frac{1}{2} \boldsymbol{\epsilon}:{ }^{4} \mathbf{Z}: \boldsymbol{\epsilon},
$$

with

$$
\boldsymbol{\epsilon}=\sum_{a \in \mathcal{A}} s_{a} \boldsymbol{\epsilon}_{a}
$$

where

$$
\sum_{a \in \mathcal{A}} s_{a}=1
$$

Here, $\boldsymbol{\epsilon}_{a}$ denotes one of the generalised strain tensors of the previous section. The fact that the sum of the weights $\left(s_{a}\right)$ equals one again guarantees that the response for infinitesimally small deformations is the same as for the conventional models (i.e. independent of the value of $a$ ). Again, if $\mathcal{A}$ contains a single value, the generalisation reduces to the conventional hyperelasticity of the previous section.

Strain tensor $\boldsymbol{\epsilon}$ can now be computed as follows:

$$
\boldsymbol{\epsilon}=\sum_{i=1}^{3} \epsilon_{i} \vec{n}_{i} \vec{n}_{i}
$$


with

$$
\epsilon_{i}=\sum_{a \in \mathcal{A}} s_{a} \epsilon_{i / a}
$$

where

$$
\epsilon_{i / a}=\left\{\begin{array}{lll}
\frac{1}{a}\left(c_{i}^{a / 2}-1\right) & \text { if } & a \neq 0 \\
\frac{1}{2} \ln \left(c_{i}\right) & \text { if } & a=0
\end{array}\right.
$$

The formulation of the $1^{\text {st }}$ Piola-Kirchhoff stress tensor follows the same construction as for those of the conventional models of the previous section, except that subscript $a$ in Eqs. (6) and (7) must be omitted and that $d_{i}$ in Eqs. (8) must be replaced by:

$$
d_{i}=\sum_{a \in \mathcal{A}} s_{a} c_{i}^{(a / 2)-1}
$$

Also the tangent stiffness tensor follows the same construction as for those of the conventional models of the previous section, except that subscript $a$ in Eqs. (11) and (12) must be omitted and that $g_{i}$ in Eqs. (13) must be replaced by:

$$
g_{i}=\sum_{a \in \mathcal{A}} s_{a}(a-2) c_{i}^{(a / 2)-2} .
$$

\subsection{Volume change dependent fusing of the strain tensors}

The third generalisation is similar to the second one, except that the weights used to gather the conventional strain tensors in one strain tensor are dependent on the volume change. In this generalisation, the strain energy density is thus again written as:

$$
W=\frac{1}{2} \boldsymbol{\epsilon}:{ }^{4} \mathbf{Z}: \boldsymbol{\epsilon}
$$

but strain tensor $\boldsymbol{\epsilon}$ is now defined as:

$$
\boldsymbol{\epsilon}=\sum_{a \in \mathcal{A}} s_{a}(J) \boldsymbol{\epsilon}_{a}
$$


where

$$
\sum_{a \in \mathcal{A}} s_{a}(J)=1
$$

Strain tensor $\boldsymbol{\epsilon}$ is computed as follows:

$$
\boldsymbol{\epsilon}=\sum_{i=1}^{3} \epsilon_{i} \vec{n}_{i} \vec{n}_{i}
$$

with

$$
\epsilon_{i}=\sum_{a \in \mathcal{A}} s_{a}(J) \epsilon_{i / a}
$$

where

$$
\epsilon_{i / a}=\left\{\begin{array}{lll}
\frac{1}{a}\left(c_{i}^{a / 2}-1\right) & \text { if } & a \neq 0 \\
\frac{1}{2} \ln \left(c_{i}\right) & \text { if } & a=0 .
\end{array}\right.
$$

\subsubsection{Stress tensors}

The expression for the $1^{\text {st }}$ Piola-Kirchhoff stress tensor is now slightly more complicated, as it can be written as:

$$
\mathbf{P}=\frac{\partial W}{\partial \mathbf{F}}=2 \mathbf{F} \cdot\left({ }^{4} \mathbf{Z}: \boldsymbol{\epsilon}: \sum_{a \in \mathcal{A}} s_{a}(J) \frac{\partial \boldsymbol{\epsilon}_{a}}{\partial \mathbf{C}}\right)+J^{4} \mathbf{Z}: \boldsymbol{\epsilon}: \sum_{a \in \mathcal{A}} \frac{\partial s_{a}}{\partial J} \boldsymbol{\epsilon}_{a} \mathbf{F}^{-c}
$$

The first term in this expression corresponds to the $1^{\text {st }}$ Piola-Kirchhoff stress tensor of the second generalisation. The second term results from the fact that the weights are made functions of the volume change. It is worth mentioning that the second term has no influence for infinitesimally small deformations because of the following:

$$
\left[\sum_{a \in \mathcal{A}} \frac{\partial s_{a}}{\partial J} \boldsymbol{\epsilon}_{a}\right]_{\mathbf{F} \approx \mathbf{I}}=\mathbf{0}
$$

The reasons for this are that $\boldsymbol{\epsilon}_{a}$ is the same for infinitesimally small deformations for any choice of $a$ and:

$$
\sum_{a \in \mathcal{A}} \frac{\partial s_{a}}{\partial J}=0
$$


Of course the second term does have an influence on the stress for large deformations. It is therefore worth to check if the part of the Cauchy stress tensor resulting from the second term is frame-indifferent and abides the balance of linear momentum (i.e. if it is symmetric). The Cauchy stress tensor resulting from the second term can be expressed as follows:

$$
\boldsymbol{\sigma}=\frac{1}{J} \mathbf{P}: \mathbf{F}^{c}=\left({ }^{4} \mathbf{Z}: \boldsymbol{\epsilon}: \sum_{a \in \mathcal{A}} \frac{\partial s_{a}}{\partial J} \boldsymbol{\epsilon}_{a}\right) \mathbf{I},
$$

which is a hydrostatic stress tensor, since the term between parentheses is an invariant scalar. Rigid body rotations clearly have no influence and symmetry is also guaranteed.

\subsubsection{Tangent stiffness tensor}

Since the tangent stiffness tensor necessary for FE computations is more involved than for the previous two generalisations, it is presented here explicitly as follows:

$$
\begin{aligned}
&{ }^{4} \mathbf{K}= \frac{\partial^{2} W}{\partial(\mathbf{F})^{2}}=2\left(\mathbf{Z}: \boldsymbol{\epsilon}: \sum_{a \in \mathcal{A}} s_{a}(J) \frac{\partial \boldsymbol{\epsilon}_{a}}{\partial \mathbf{C}} \mathbf{I}\right)^{[14]}+2 J \mathbf{F} \cdot\left({ }^{4} \mathbf{Z}: \boldsymbol{\epsilon}: \sum_{a \in \mathcal{A}} \frac{\partial s_{a}}{\partial J} \frac{\partial \boldsymbol{\epsilon}_{a}}{\partial \mathbf{C}}\right) \mathbf{F}^{-c}+ \\
& 4 \mathbf{F} \cdot\left({ }^{4} \mathbf{Z}: \boldsymbol{\epsilon}: \sum_{a \in \mathcal{A}} s_{a}(J) \frac{\partial^{2} \boldsymbol{\epsilon}_{a}}{\partial(\mathbf{C})^{2}} \cdot \mathbf{F}^{c}\right)^{[34]}+2 J \mathbf{F}^{-c} \mathbf{F} \cdot\left({ }^{4} \mathbf{Z}: \boldsymbol{\epsilon}: \sum_{a \in \mathcal{A}} \frac{\partial s_{a}}{\partial J} \frac{\partial \boldsymbol{\epsilon}_{a}}{\partial \mathbf{C}}\right)+ \\
& J^{4} \mathbf{Z}: \boldsymbol{\epsilon}:\left(\sum_{a \in \mathcal{A}}\left(\frac{\partial s_{a}}{\partial J}+J \frac{\partial^{2} s_{a}}{\partial J^{2}}\right) \boldsymbol{\epsilon}_{a} \mathbf{F}^{-c} \mathbf{F}^{-c}-\sum_{a \in \mathcal{A}} \frac{\partial s_{a}}{\partial J} \boldsymbol{\epsilon}_{a}\left(\mathbf{F}^{-1} \mathbf{F}^{-1}\right)^{[34,23,12]}\right)+ \\
&\left({ }_{2} \mathbf{F} \cdot\left(\sum_{a \in \mathcal{A}} s_{a}(J) \frac{\partial \boldsymbol{\epsilon}_{a}}{\partial \mathbf{C}}\right)^{[13,24]}+J \mathbf{F}^{-c} \sum_{a \in \mathcal{A}} \frac{\partial s_{a}}{\partial J} \boldsymbol{\epsilon}_{a}\right):{ }^{4} \mathbf{Z}: \\
&\left(J \sum_{a \in \mathcal{A}} \frac{\partial s_{a}}{\partial J} \boldsymbol{\epsilon}_{a} \mathbf{F}^{-c}+2\left(\sum_{a \in \mathcal{A}} s_{a}(J) \frac{\partial \boldsymbol{\epsilon}_{a}}{\partial \mathbf{C}} \cdot \mathbf{F}^{c}\right)^{[34]}\right)
\end{aligned}
$$

\section{Finite element framework}

In this section, the formulation of a finite element (FE) framework for the aforementioned constitutive models is briefly discussed. Body forces are excluded. 


\subsection{Material basis and global basis}

First, it is important to mention that all tensors in the previous two sections are expressed in terms of the material basis (which may vary based on reference location $\vec{X}$ ), whereas all tensors in the FE framework are expressed in the global basis. Tensors in the global basis are distinguished by a tilde (e.g. $\tilde{\mathbf{F}}$ ) in order to distinguish them from their counterparts defined in the material basis. Although this distinction is not necessary for isotropy, it is needed for transverse isotropy and orthotropy. Deformation-independent rotation tensor $\mathbf{R}_{0}(\tilde{\vec{X}})$ is used to rotate a spatial vector in the global basis to the material basis as follows:

$$
\vec{X}=\mathbf{R}_{0} \cdot \tilde{\vec{X}}
$$

or vice versa as follows:

$$
\tilde{\vec{X}}=\mathbf{R}_{0}^{c} \cdot \vec{X}
$$

This implies that the deformation gradient tensor in the material basis is related to that in the global basis as follows:

$$
\mathbf{F}=\mathbf{R}_{0} \cdot \tilde{\mathbf{F}} \cdot \mathbf{R}_{0}^{c}
$$

\subsection{Interpolation and potential energy}

As often considered in FE computations, the displacement field is interpolated using shape functions, which are stored in column $\underline{N}(\tilde{\vec{X}})$, where each shape function is associated with an FE node. The original location vectors and displacement vectors at the FE nodes are stored in columns $\underline{\tilde{\vec{X}}}$ and $\underline{\vec{u}}$, respectively, which are both of the same length as $\underline{N}(\tilde{\vec{X}})$. The fields of displacement vectors and deformation gradient tensors can now be expressed in the global basis as follows:

$$
\begin{aligned}
& \tilde{\overrightarrow{\vec{u}}}(\tilde{\vec{X}})=\underline{N}^{T}(\tilde{\vec{X}}) \underline{\overrightarrow{\vec{u}}}, \\
& \tilde{\mathbf{F}}(\tilde{\vec{X}})=\mathbf{I}+\left(\tilde{\vec{\nabla}}_{0} \underline{N}^{T} \underline{\overrightarrow{\vec{u}}}\right)^{c} .
\end{aligned}
$$


The solution of an FE simulation with the aforementioned hyperelastic models can be expressed as the minimisation of the potential energy as follows:

$$
\underline{\overrightarrow{\vec{u}}}^{*}=\underset{\underline{\tilde{u}}}{\operatorname{argmin}} \int_{V_{0}} W(\tilde{\mathbf{F}}(\underline{\tilde{\vec{u}}})) d V_{0}-\underline{\tilde{f}}_{\text {ext }}^{\underline{\sim}} \cdot \underline{\tilde{\vec{u}}},
$$

where $\underline{\tilde{f}}_{\text {ext }}^{\tilde{g}}$ denotes the column with forces externally applied at the FE nodes in the global basis and $V_{0}$ denotes the undeformed volume.

\subsection{Fermat's theorem and its linearisation}

The minimisation of the potential energy is commonly computed using Fermat's theorem and applying Newton's method, since good initial guesses are generally known. Fermat's theorem applied to the aforementioned potential energy yields the following equation:

$$
\int_{V_{0}} \frac{\partial W}{\partial \mathbf{F}}: \frac{\partial \mathbf{F}^{c}}{\partial \tilde{\mathbf{F}}}: \frac{\partial \tilde{\mathbf{F}}^{c}}{\partial \underline{\vec{u}}} d V_{0}=\underline{\vec{f}}_{e x t},
$$

where

$$
\begin{aligned}
& \frac{\partial \mathbf{F}^{c}}{\partial \tilde{\mathbf{F}}}=\left(\mathbf{R}_{0} \mathbf{R}_{0}^{c}\right)^{[24]}, \\
& \frac{\partial \tilde{\mathbf{F}}^{c}}{\partial \underline{\overrightarrow{\tilde{u}}}}=\tilde{\vec{\nabla}}_{0} \underline{N} \mathbf{I} .
\end{aligned}
$$

Eq. (47) can then also be written as:

$$
\underline{\tilde{\vec{f}}}_{\text {int }}(\underline{\tilde{\vec{u}}})=\underline{\overrightarrow{\vec{f}}}_{\text {ext }},
$$

with

$$
\underline{\tilde{\vec{f}}}_{i n t}(\underline{\tilde{\vec{u}}})=\int_{V_{0}} \tilde{\vec{\nabla}}_{0} \underline{N} \cdot\left(\mathbf{R}_{0} \mathbf{R}_{0}\right)^{[14]}: \mathbf{P}(\underline{\tilde{\vec{u}}}) d V_{0} .
$$

The $1^{\text {st }}$-order Taylor expansion of the force equilibrium in Eq. (50) necessary for the application of Newton's method can then be expressed as follows:

$$
\underline{\vec{f}}_{i n t}\left(\underline{\overrightarrow{\vec{u}}}_{e}\right)+\underline{\underline{\mathbf{K}}}\left(\underline{\tilde{\vec{u}}}_{e}\right) \cdot d \underline{\overrightarrow{\vec{u}}}=\underline{\vec{f}}_{e x t},
$$


where $\underline{\overrightarrow{\vec{u}}}_{e}$ denotes the current estimate of $\underline{\overrightarrow{\vec{u}}}^{*}$ and $d \underline{\overrightarrow{\vec{u}}}$ the correction to $\underline{\overrightarrow{\vec{u}}}_{e}$ that is to be computed. Matrix of $2^{\text {nd }}$-order stiffness tensors $\underline{\underline{\mathbf{K}}}$ can furthermore be computed as:

$$
\underline{\underline{\tilde{\mathbf{K}}}}\left(\underline{\tilde{\vec{u}}}_{e}\right)=\int_{V_{0}} \tilde{\vec{\nabla}}_{0} \underline{N} \cdot\left(\mathbf{R}_{0} \mathbf{R}_{0}\right)^{[14]}:{ }^{4} \mathbf{K}\left(\underline{\tilde{\vec{u}}}_{e}\right):\left(\mathbf{R}_{0} \mathbf{R}_{0}^{c}\right)^{[24]} \cdot \tilde{\vec{\nabla}}_{0} \underline{N}^{T} d V_{0},
$$

where the expressions for ${ }^{4} \mathbf{K}$ are given in the previous sections, similar as the expressions for $\mathbf{P}$.

The FE implementation used to generate the results in the next section employs a Total Lagrangian procedure and trilinear hexagonal elements (with eight quadrature points) and an isoparametric mapping.

\section{Results}

In the current section the conventional hyperelasticity based on the SethHill strain tensors is compared to the three generalisations and the identification of the new material parameters is presented. In the first subsection, the conventional hyperelasticity is discussed. In the second subsection, some results for the three generalisations are presented and compared to the conventional hyperelasticity. In the third subsection, the identification is discussed for isotropy. The fourth subsection also discusses the identification, but focuses on orthotropy. The identified parameters are also propagated for a typical orthotropic test case.

In case of isotropy, a Young's modulus of 1 and a Poisson's ratio of 0.3 are used. Young's moduli $Y_{1}=1, Y_{2}=2, Y_{3}=3$, Poisson's ratios $\nu_{12}=$ $\nu_{13}=\nu_{23}=0.3$ and shear moduli $G_{12}=0.5, G_{13}=1, G_{23}=1.5$ are used for orthotrpy. The identification presented below only focuses on the identification of the new material parameters. Hence, the Young's moduli, Poisson's ratios and shear moduli are assumed to already be identified.

\subsection{Conventional hyperelasticity}

The only unconventional material parameter in the conventional hyperelasticy based on the Seth-Hill strain tensors is $a$. In Fig. 2, some Cauchy stress-deformation responses are presented for different values of $a$ (ranging from -3 to 3 ) if the deformation is fully prescribed. In case of uniaxial deformation (top-left and centre-left diagrams), only the Hencky strain tensor $(a=0)$ behaves such that the diagonal component of the Cauchy stress tensor associated with the direction in which deformation takes place (i) tends 


$$
--a=-3 \longrightarrow a=0 \cdots \cdots \cdots \cdot a=3-a=-2.5,-2,-1.5,-1,-0.5,0.5,1,1.5,2,2.5
$$
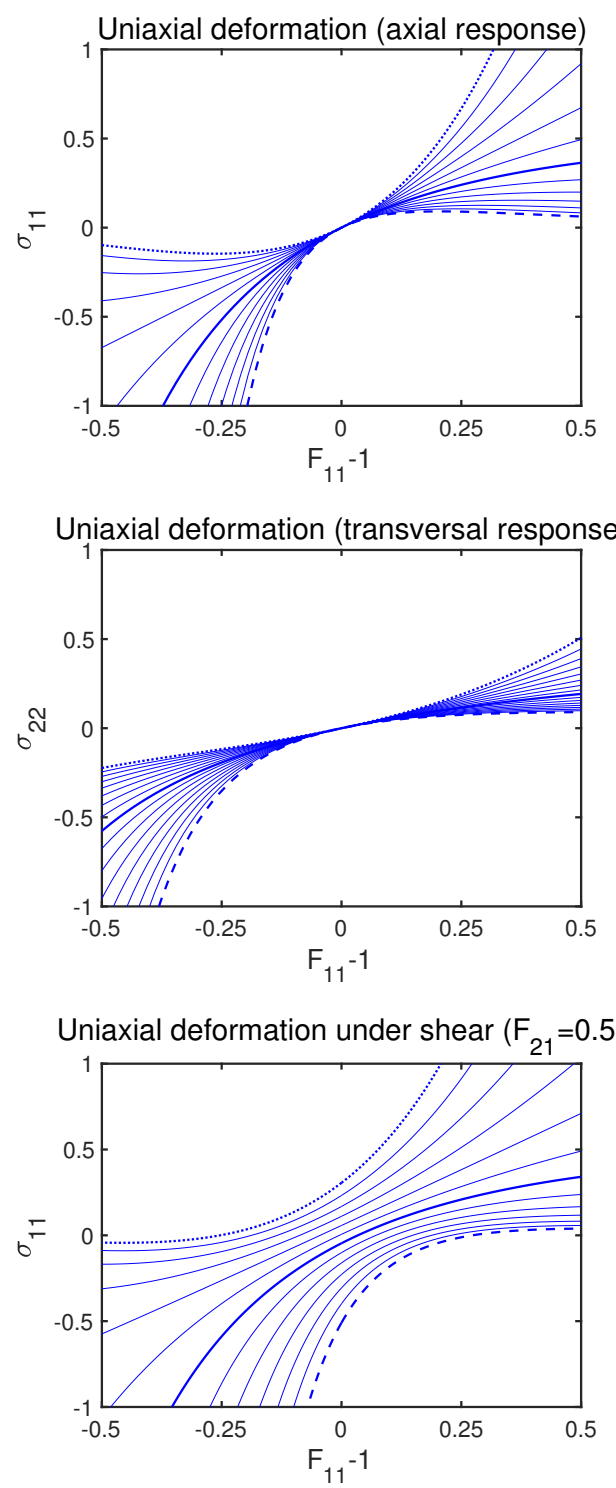
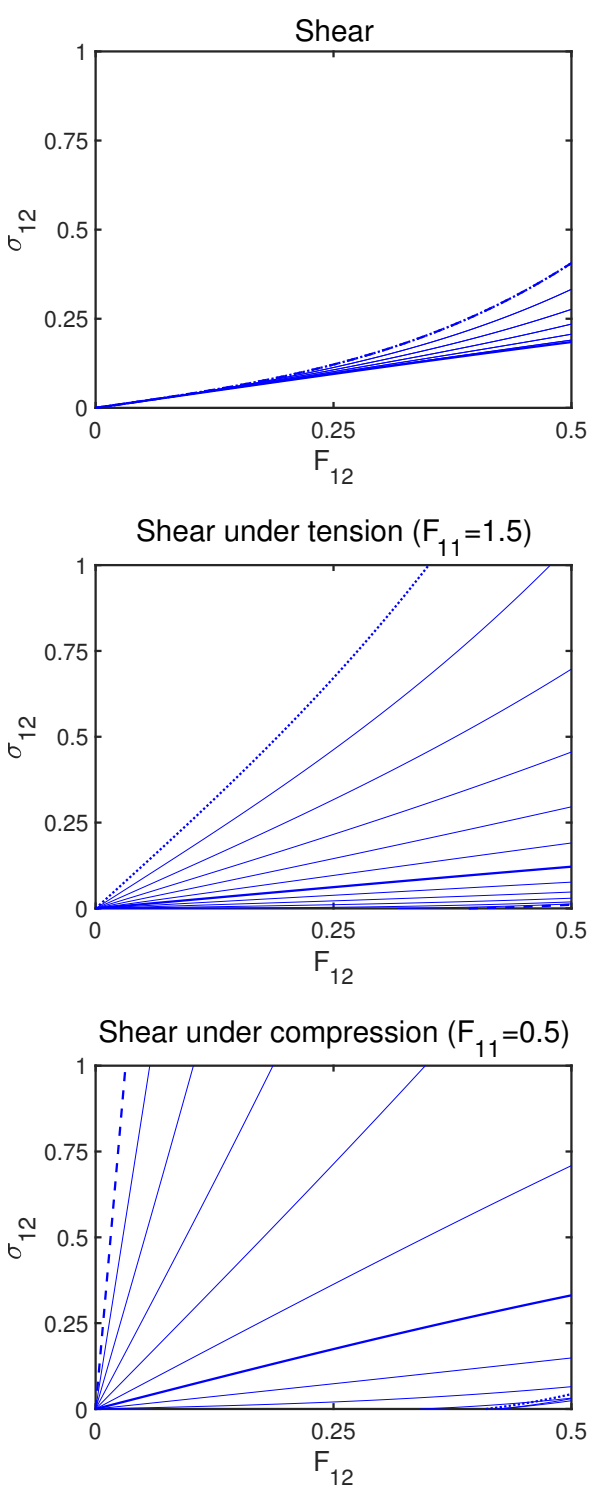

Figure 2: Conventional hyperelasticity: Cauchy stress-deformation responses for homogeneous deformations with different strain tensors, given by parameter $a$ (see Eq. (2)). Unless mentioned otherwise, all components of the deformation gradient tensor are according to $\mathbf{F}=\mathbf{I}$. 


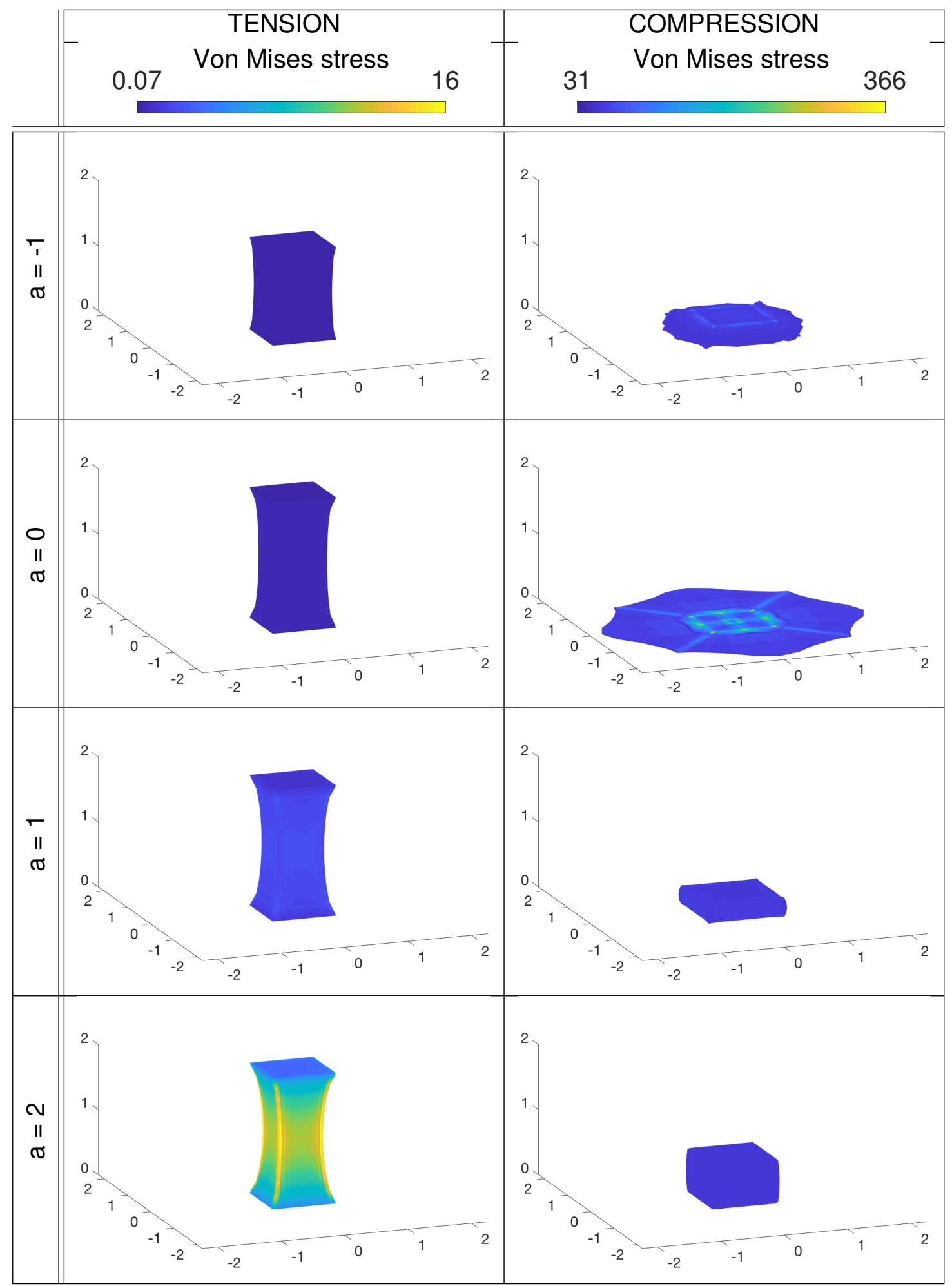

Figure 3: Conventional hyperelasticity: unit cubes exposed to tension (left column) and compression (right column) for different strain tensors, given by parameter $a$ (see Eq. (2)). The deformations are not scaled and presented on the same scale. 

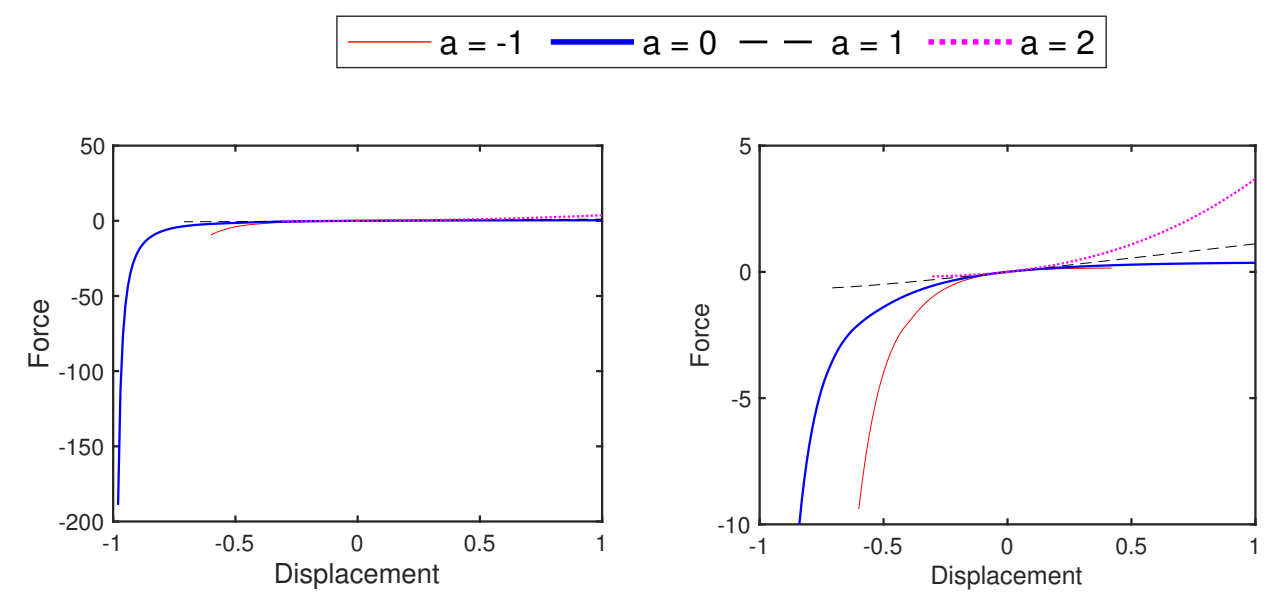

Figure 4: Conventional hyperelasticity: the force-displacement responses of the unit cubes of Fig. 3 on two different scales.

to $-\infty$ if the volume tends to 0 and (ii) monotonically increases for an increase of the deformation. This means that the slope of the response for all other values of $a$, either will not tend to $-\infty$ for large compressive deformations or will be 0 for some deformation. Both cases are not very physical and may result in problems in FE computations; stiffness matrices may become singular, for instance due to inverted FEs.

From the shear responses (right diagrams in Fig. 2), it also becomes clear why the Hencky strain tensor is often used. After all, the slope of the Cauchy shear stress remains positive even if relatively large compressive or tensile deformation is applied. The slope only becomes negative if substantially large compressive deformations are applied (not presented here).

To illustrate in more detail why the Hencky strain tensor is often used, results of FE computations of the compression and elongation of unit cubes are presented in Figs. 3 and 4 for four values of $a$. The unit cubes are entirely compressed and elongated with an elongation factor of two (each in 100 increments) by fully prescribing all displacements on the top and bottom surfaces (i.e. dilatation of the top and bottom surfaces is suppressed). In case FE nodes penetrate the virtual planes of the top and bottom surfaces during the compression test, the FE nodes are placed back to the virtual planes to prevent snap-through. These FE nodes in contact are free to slide over the virtual planes. 


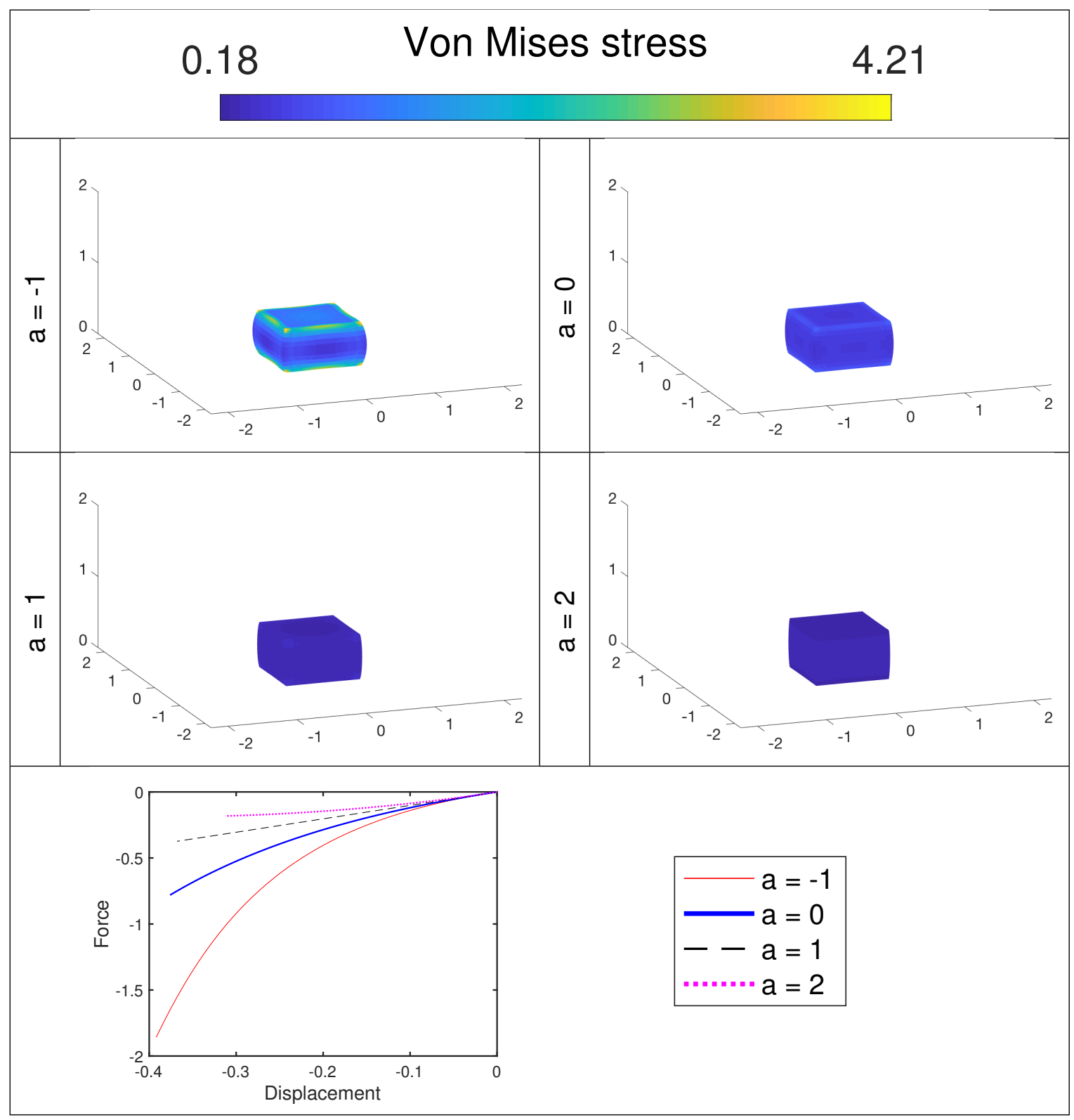

Figure 5: Conventional hyperelasticity: unit cubes exposed to compression with friction for different strain tensors, given by parameter $a$ (see Eq. (2)). The deformations are not scaled and presented on the same scale. 
Only $\frac{1}{8}$ of the model is considered thanks to symmetry boundary conditions. 125 equally sized hexagonal FEs are used to discretise the considered part of the model (resulting in element dimensions of $0.1 \times 0.1 \times 0.1$ ). If convergence is not found for an increment, the computation is stopped (convergence is based on the relative force residual and the tolerance is set to $10^{-5}$ ). The results show that conventional hyperelasticity with $a=0$ is indeed most robust. All FE computations, except the one with $a=-1$, are able to converge in extension with an elongation factor of two.

In another set of FE computations, the same unit cubes with the same strain tensors as in Figs. 3 and 4 are considered, but they are exposed to frictional compression. In other words, the cubes are compressed whilst dry friction (according to Coulomb's friction model with a friction coefficient of 0.08) prevents the top and bottom surfaces from freely dilating. The results are presented in Fig. 5 and again show a substantial robustness for the Hencky strain tensor, although the Almansi strain tensor performs slightly better for this test case.

Although the results have so far mainly been analysed in terms of robustness, the deformations, reaction forces and stress fields (presented in the figures in terms of Von Mises's stress criterion for the Cauchy stress tensor) of course also depend on the value of $a$. During extension of the unit cubes for instance (left images in Fig. 3), the amount of contraction increases for an increase of $a$, whilst the Von Mises stress increases to localise at the edges for an increase of $a$. The slope of the reaction forces during extension furthermore increases for an increase of $a$.

\subsection{The three generalisations}

The idea behind the generalisations is to take advantage of the robustness of the Seth-Hill strain tensors with values of $a \leq 0$ in compression, whilst at the same time incorporate Seth-Hill strain tensors with values of $a>0$ to ensure that the slope of the stress-deformation response continues to grow in tension. In this way, the generalisations provide freedom to tailor the stressdeformation/force-displacement responses and/or the local stress-strain fields in the nonlinear regime, whilst the robustness is increased or remains the same.

The first set of results presented in Fig. 6 illustrate that the generalisations indeed yield material responses that cannot be generated with the conventional hyperelasticity. The results for the first generalisation (in which 


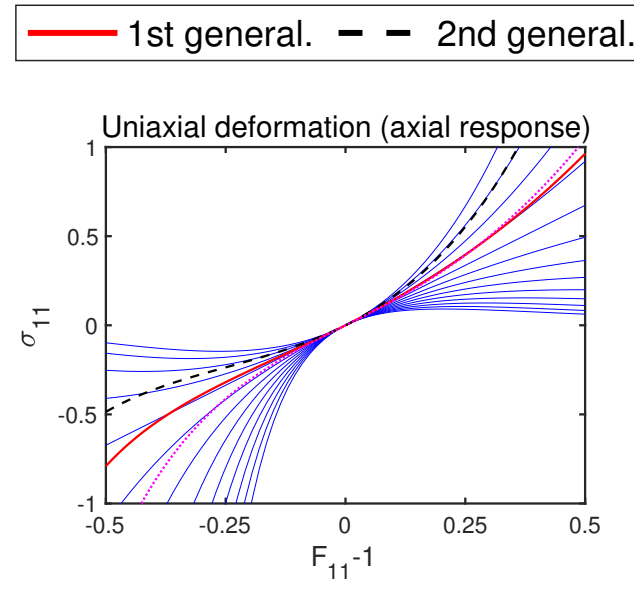

....... 3rd general. — conventional
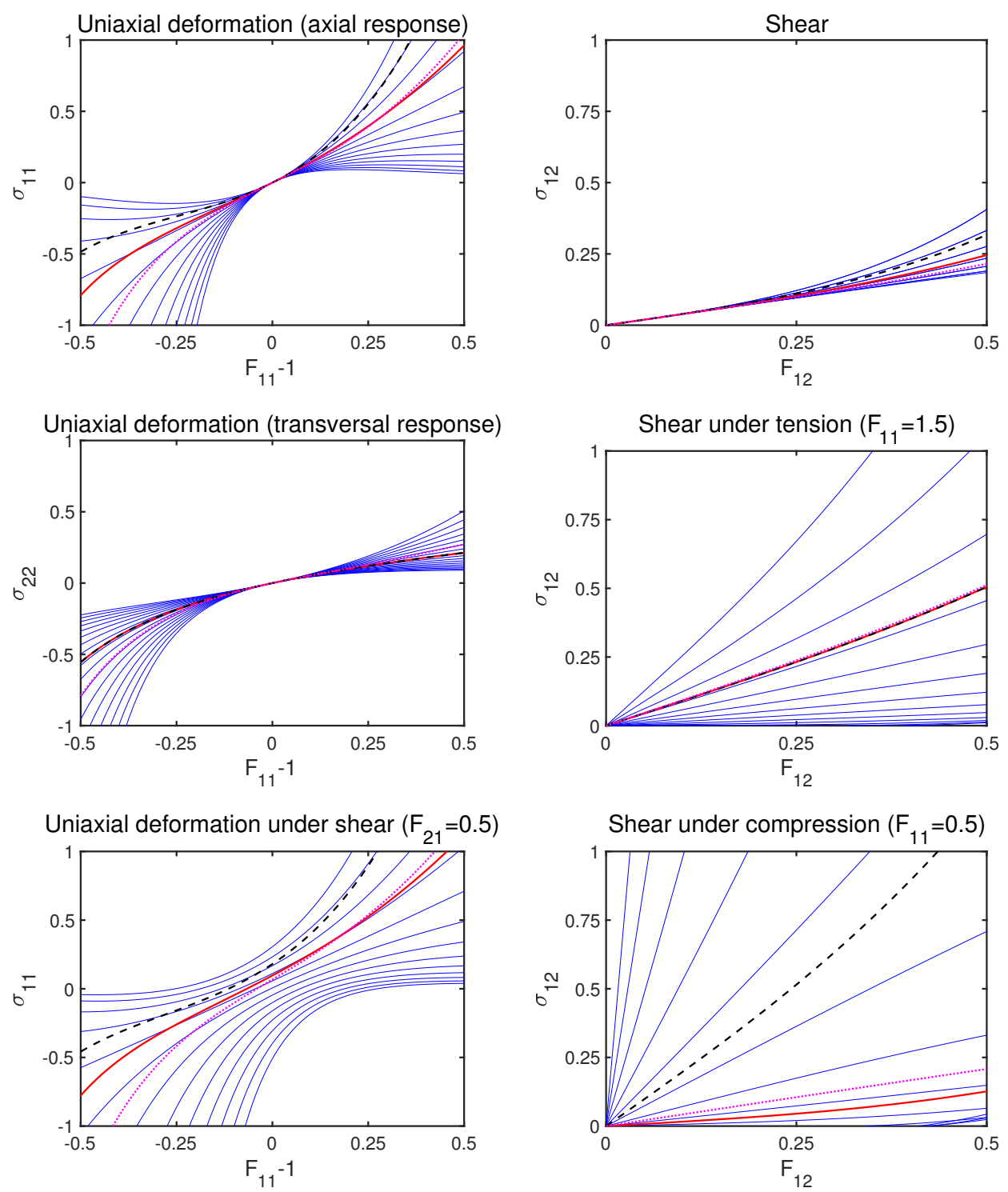

Figure 6: The three generalisations: Cauchy stress-deformation responses for homogeneous deformations compared to those of the conventional hyperelasticity. Unless mentioned otherwise, all components of the deformation gradient tensor are according to $\mathbf{F}=\mathbf{I}$. 


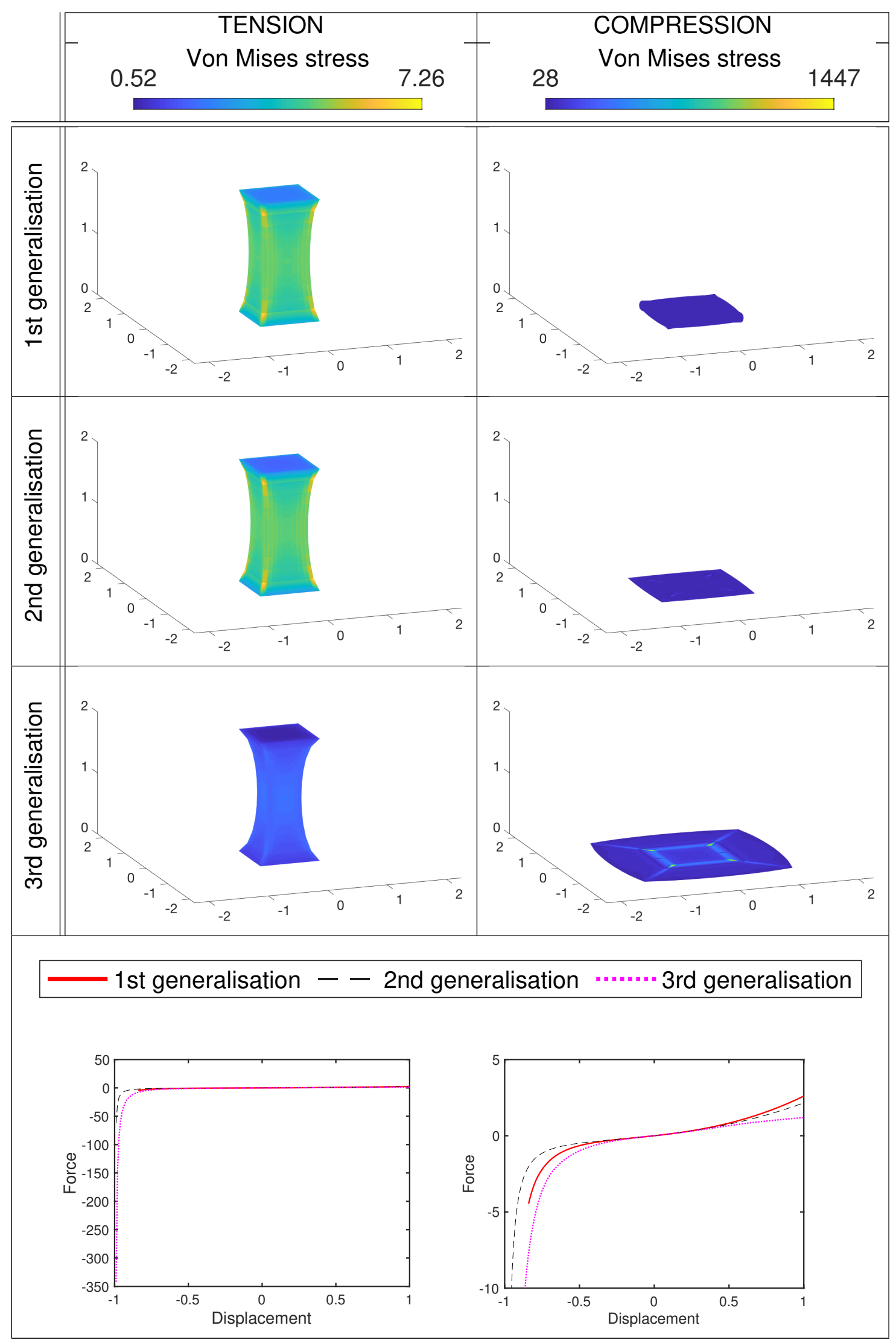

Figure 7: The three generalisations: unit cubes exposed to tension (left column) and compression (right column). Bottom row: the force-displacement responses on two different scales. 


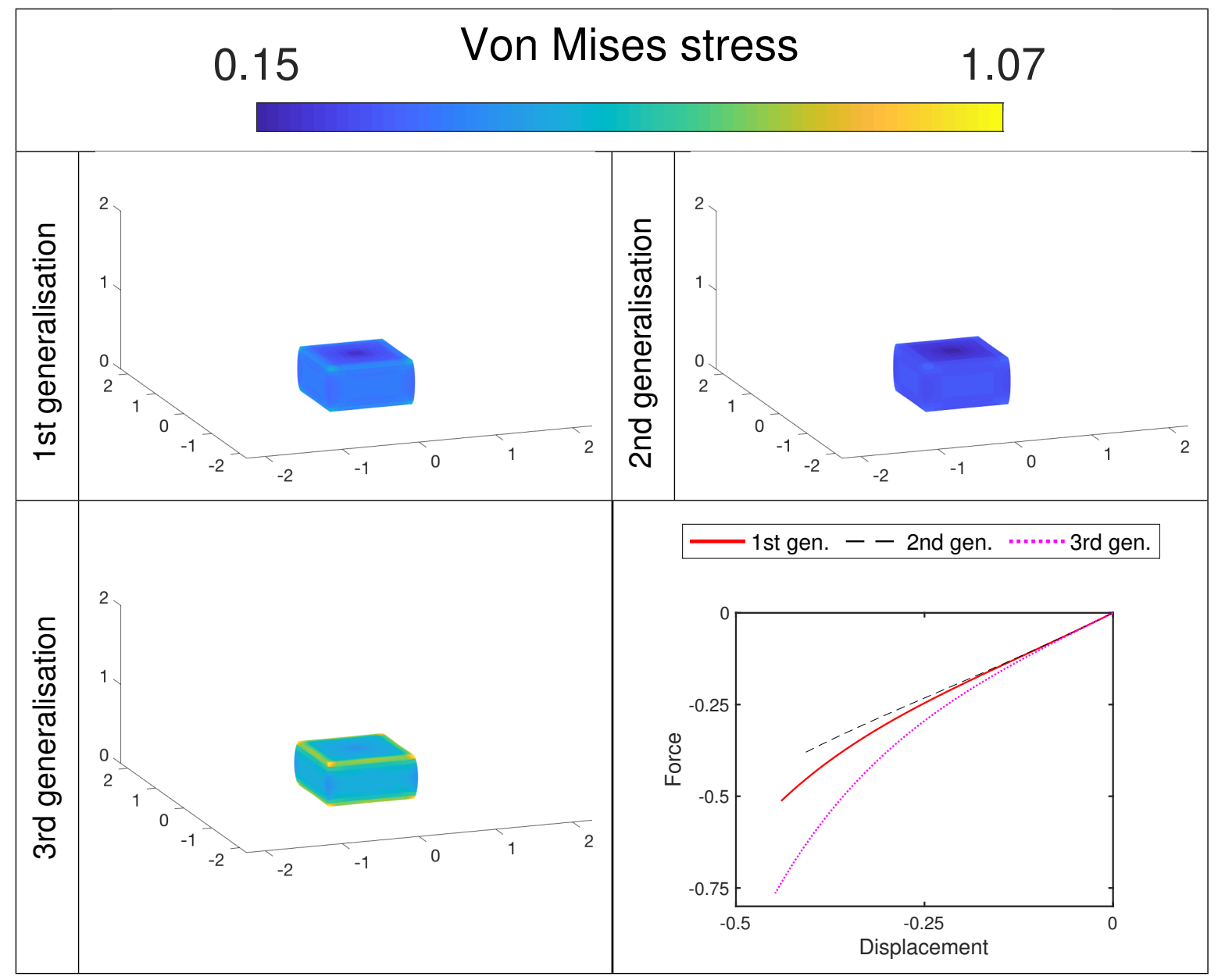

Figure 8: The three generalisations: unit cubes exposed to compression with friction. The deformations are not scaled and presented on the same scale.

the strain energy densities are summed in a weighted average sense) are associated with parameters $r_{0}=\frac{1}{3}$ and $r_{2}=\frac{2}{3}$ (where the subscript indicates the value of $a$ ). The results for the second generalisation (in which the conventional strain tensors are summed in a weighted average sense) are associated with parameters $s_{0}=\frac{1}{3}$ and $s_{2}=\frac{2}{3}$. The results for the third generalisation are associated with the following volume change dependent weights:

$$
\begin{aligned}
& s_{a_{1}}(J)=\frac{-\tan ^{-1}\left(p_{1}\left(J-p_{2}\right)\right)}{\pi}+0.5, \\
& s_{a_{2}}(J)=1-s_{a_{1}}(J)
\end{aligned}
$$

where $a_{1}$ and $a_{2}$ denote the values for $a$ for the two strain tensors and $p_{1}$ and $p_{2}$ denote material parameters that describe functions $s$. For the results in 
Fig. 6 (and also for the other results in the current subsection), their values are set to $a_{1}=0, a_{2}=2, p_{1}=2$ and $p_{2}=1$. We indeed observe that none of the mechanical responses of the three generalisations can yield from the conventional hyperelasticity based on the generalised strain tensors.

The same FE computations are now considered as for the conventional hyperelasticity of the previous subsection. The same unconventional material parameters are used as mentioned in the previous paragraph. The results in case the displacements of the top and bottom surfaces are fully prescribed are presented in Fig. 7. Substantially larger compressive deformations are obtained than for most conventional hyperelastic models and the contraction during elongation is substantially larger, even though the Hencky strain tensor is present in all three generalisations.

The results for frictional compression of the unit cubes are presented in Fig. 8. Most interesting to observe is that the force-displacement curves are significantly more linear than for the conventional hyperelasticity and again convergence is obtained for a larger compressive deformation than for the conventional hyperelasticity.

\subsection{Identification for isotropy}

The previous two subsections have shown that the generalisations are indeed different from the conventional hyperelasticity based on generalised strain tensors and that they offer more flexibility to tune stress-strain responses and local stress and strain fields. The current and the next subsections focus on the identification of the new parameters; the current one for isotropy and the next one for orthotropy. In both subsections, a virtual tensile test and compression test are performed to provide reference forcedisplacement curves in tension and compression. The new parameters of the different hyperelastic models are to be identified based on these reference data. Due to the nonlinearity of the models, (small) inverse models are required.

The setups and final results of the virtual tensile test and compression test for isotropy are presented in Fig. 9. They are computed using the second generalisation with $s_{-1}=0.25, s_{0.5}=0.25$ and $s_{3.5}=0.5$ (and again a Young's modulus of 1 and a Poisson's ratio of 0.3 are used). The dimensions are presented in Fig. 9 and in its caption. All displacements of the top and bottom FE nodes are prescribed in the tensile test. A final vertical displacement of the top FE nodes of 5 is prescribed in 100 increments. The 
computation of the virtual tensile test uses 1,920 FEs; 12 in horizontal direction, 40 in vertical direction and 4 in out-of-plane of direction of Fig. 9. The compression test is the same as the frictional compression tests discussed in the previous two subsections, with the same friction coefficient and the same number of FEs (albeit the FE dimensions differ due to the different model domain). Note that a final vertical displacement of -0.8 is prescribed in 100 increments (but only the first 62 increments converge for this case).

\section{Identification approach}

True inverse models are needed to identify the new parameters, but full inverse models of both tests are computationally demanding. A single material point exposed to homogenous deformation without shear contributions is therefore used for the inverse model of the tensile test. If we compare the force-displacement response predicted by the tensile test and by the single material point for the true parameters (i.e. $s_{-1}=0.25, s_{0.5}=0.25$ and $s_{3.5}=0.5$ ), a small and acceptable discrepancy occurs (bottom-left diagram of Fig. 9). Note however that the exact parameters cannot be recovered.

If a single material point exposed to homogeneous deformation without friction and shear contributions would also be used for the inverse model of the frictional compression test, a larger discrepancy occurs than for the tensile test (dashed red line in the bottom-right diagram of Fig. 9). The employed inverse model of the frictional compression test therefore consists of a single FE with frictional contact (which represents $\frac{1}{8}$ of the domain due to symmetry boundary conditions), although this is computationally more demanding than a single material point. The response of the inverse model for the true parameters is clearly sufficiently close to that of the full compression test, albeit not perfect (dotted black line in the bottom-right diagram of Fig. 9).

The difference between the reference responses and the responses of the inverse models is minimised with respect to the new parameters (that are to be identified) with a nonlinear conjugate-gradient/steepest-descent approach (see e.g. Nocedal \& Wright (2006)). The approach includes a line search and estimates the steepest descent direction using finite differences. Initially, the search directions are the conjugate-gradient directions (where the Polak-Ribiere measure is used to weigh the previous search direction with the current steepest-descent direction (Polak \& Ribiere, 1996), (Nocedal \& Wright, 2006)). After the conjugate-gradient approach has converged, the steepest-descent directions are employed as the search directions. 

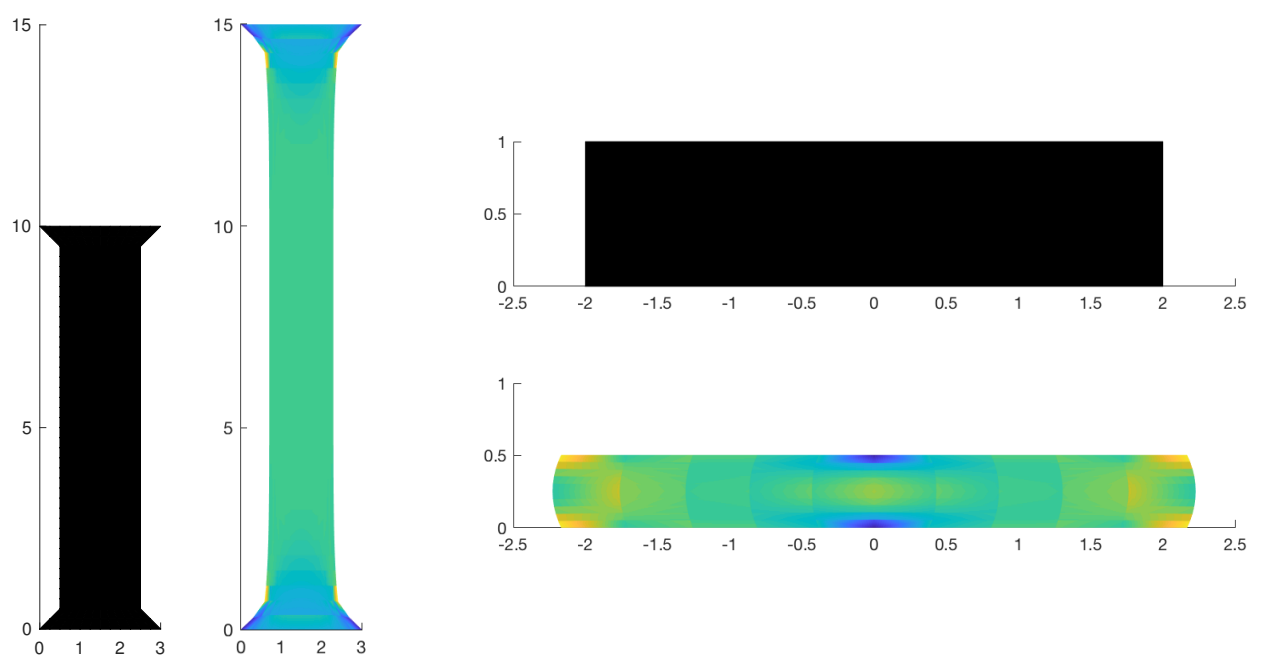

\section{full test --1 material point $\cdots \cdots \cdot 1$ finite element}
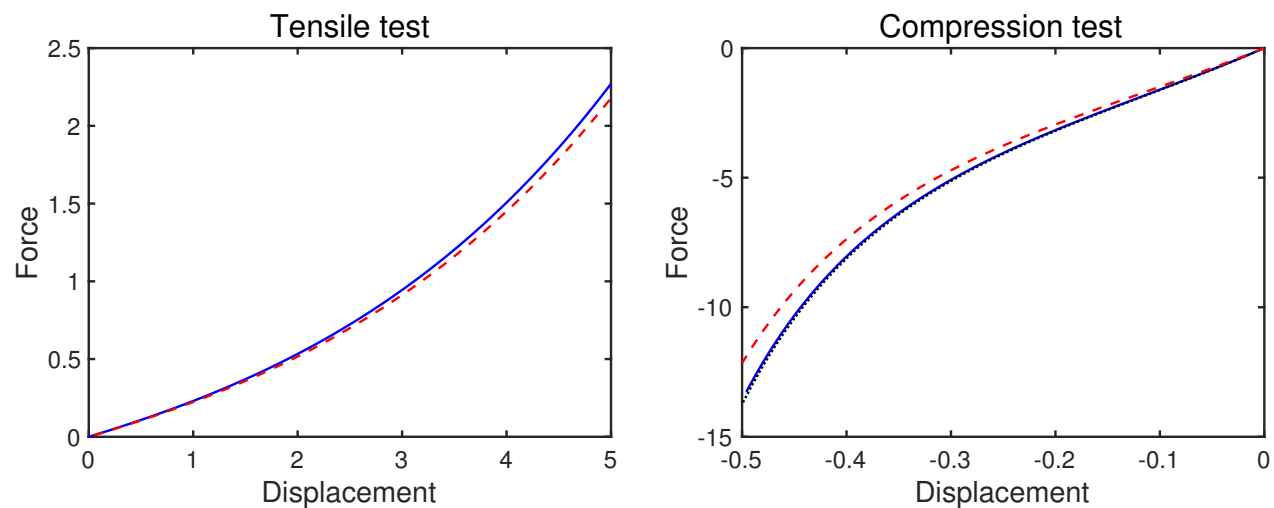

Figure 9: Identification for isotropy. Top: full tensile and compression setups as reference. Bottom: force-displacement reference curves and those a single material point and a single element for the reference parameters.

An issue of the minimisation approach is that the algorithm will propose parameter values for which the inverse models may not converge until the final elongation or compression of the reference cases is reached. Parameter values may also be proposed that give highly unphysical volume changes. In 
order to omit such sets of parameter values, the objective function is set to $\infty$ if a stiffness matrix in the inverse models is poorly conditioned or if $J<0.2$ or $J>3$ is observed in a quadrature point of the inverse models.

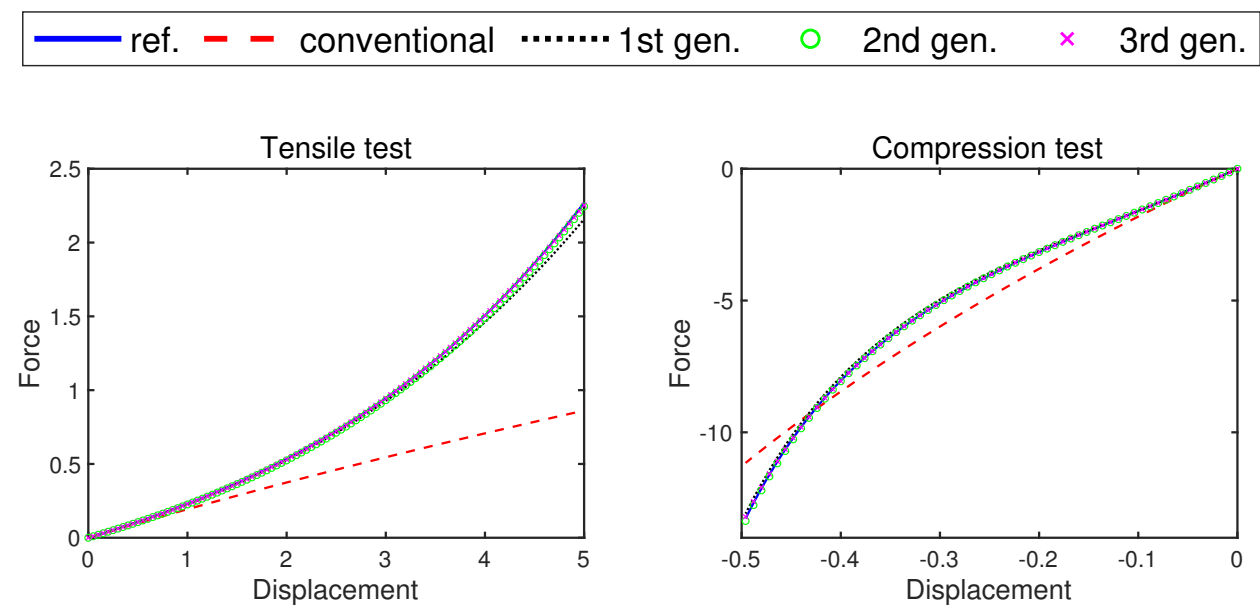

Figure 10: Identification for isotropy: the inverse model responses for the identified parameters with two strain tensors, together with the reference responses (blue).

\section{Results}

In the conventional hyperelasticity (i.e. in the proposed generalisations with a single strain tensor), only one parameter, $a$, governs the nonlinear response. The aforementioned minimisation yields a value of $a=0.76$ in this case. If each generalisation consists of two strain tensors, the identified values for the three generalisations are $r_{-0.44}=0.27$ and $r_{2.51}=0.73$ for the first generalisation, $s_{-0.66}=0.42$ and $s_{3.36}=0.58$ for the second generalisation, and $a_{1}=-0.46, a_{2}=3.49 p_{1}=0.18, p_{2}=0.17$ according to Eqs. (54) and (55) for the third generalisation. The inverse model responses associated with these values are presented in the top diagrams of Fig. 10 together with the reference data. The results are clearly sufficiently accurate if the generalisations only contain two strain tensors. We therefore do not investigate the generalisations for more than two strain tensors.

Interesting to observe in Fig. 10 is that the second generalisation with two strain tensors given by parameter values $s_{-0.66}=0.42$ and $s_{3.36}=0.58$ is highly accurate, whereas the reference results are created with the same 
generalisation, except that three strain tensors are used. Without going in more detail in this study, this may indicate that the use of more than two strain tensors does incorporate more information in the material description and/or that shear experiments can be included to provide more certainty for the identification. Another interesting observation is that the values of $p_{1}$ and $p_{2}$ for the third generalisation are substantially small; indicating that the minimisation has largely removed the volume change dependency in the third generalisation. This is to be expected as the reference data were generated using the second generalisation which lacks volume change depending weights.

\subsection{Identification for orthotropy and propagation for an ellipsoidal tube}

The identification of the new material parameters is again discussed in the current subsection, except that (i) an orthotropic material is considered, (ii) the reference responses are created using the third generalisation and (iii) the identified parameter sets are propagated for a typical orthotropic test case.

The same tensile test and compression test as in the previous subsection are used to create the measurements, using the third generalisation with unconventional parameter values $a_{1}=0, p_{1}=2, p_{2}=0.75$ and $a_{2}=4$. The aforementioned conventional parameter values of $Y_{1}=1, Y_{2}=2, Y_{3}=$ 3 for the Young's moduli, $\nu_{12}=\nu_{13}=\nu_{23}=0.3$ for the Poisson's ratios and $G_{12}=0.5, G_{13}=1, G_{23}=1.5$ for the shear moduli are employed. The conventional parameter values are considered to be already identified. Note that the horizontal direction in Fig. 9 corresponds to the first principle direction of the orthotropic model, the out-of-plane direction to the second principle direction of the orthotropic model and the vertical direction to the third principle direction of the orthotropic model.

\section{Identification results}

The aforementioned minimisation procedure yields the following results. In case of conventional hyperelasticity, a value of $a=0.87$ is identified. For the three generalisations with two strain tensors, values of $r_{1.65}=0.94$ and $r_{-0.65}=0.06$ are identified for the first generalisation, $s_{-0.09}=0.57$ and $s_{4.05}=0.43$ for the second generalisation and $a_{1}=-0.04, p_{1}=1.65$, $p_{2}=0.74$ and $a_{2}=4.36$ for the third one using Eqs. (54) and Eq. (55).

The inverse responses for these identified parameter values are presented in Fig. 11 together with the reference responses. The conventional hypere- 
lasticity with $a=0.87$ clearly performs the worst, as the responses are not able to pick up the trends of the reference data. The first and the second generalisation both perform similarly; their responses match the reference responses much better than the conventional hyperelasticity, but they are not perfect. Although different values are identified for the parameters of the third generalisation than those used to create the reference data, the responses match the reference data sufficiently accurate. This shows that the third generalisation is truly different than the first two generalisations (and hence, potentially useful).

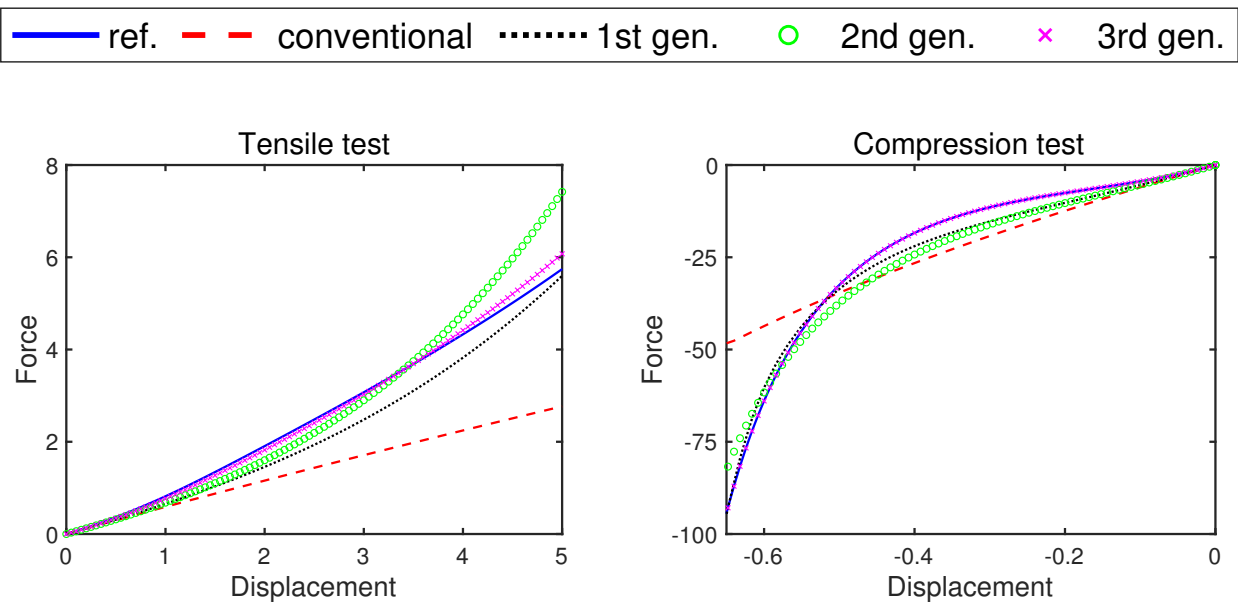

Figure 11: Identification for orthotropy: the inverse model responses for the identified parameters with two strain tensors, together with the reference responses (blue).

The parameters of the first and second generalisation are also identified in case they contain three strain tensors. The identified parameter values are $r_{-0.56}=0.07, r_{1.24}=0.15$ and $r_{2.30}=0.78$ for the first generalisation and $s_{-0.17}=0.63, s_{0.72}=-0.21$ and $s_{3.63}=0.58$ for the second generalisation. The associated inverse model responses are not presented in Fig. 11, as they are substantially similar to those in case two strain tensors are employed. This again seems to indicate that incorporating more than two strain tensors in the first two generalisations does not yield different material responses. 


\section{Propagation}

To investigate the implications of the different hyperelastic descriptions with the previously identified parameter values for a typical orthotropic test case, the focus is now on the elliptical tube of Fig. 12. The tube has a length of 1.00 and the inner cross-sectional dimensions are $R_{i n / 1}=0.075$ and $R_{\text {in } / 2}=0.15$, whilst the outer cross-sectional dimensions are $R_{\text {out } / 1}=0.12$ and $R_{\text {out } / 2}=0.20$. The second and third principle materials directions are tangential to the tube's surface and they are oriented with a $45^{\circ}$ difference with respect to the tube's axial direction (see Fig. 12). The first material direction is normal to the tube's surface.
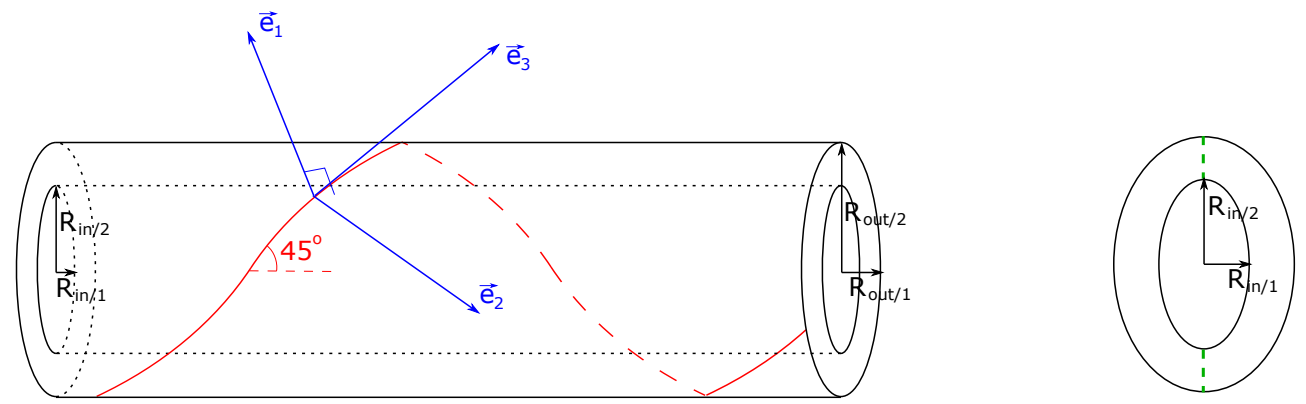

Figure 12: Propagation of unconventional material parameters for orthotropy: the material orientation in the tube and some geometrical measures.

The tube is elongated in axial direction until an elongation factor of 1.6 is reached in 100 increments. Only the axial displacements of the FE nodes at the right end in Fig. 12 are prescribed. The axial displacements of the FE nodes at the left end are also prescribed. The displacements of the FE nodes at the dotted green line in the right image of Fig. 12, at the left end of the tube, are restrained in the in-plane direction perpendicular to the green line in order to avoid twisting of the tube's cross section at the left end as much as possible. The other in-plane displacement of one of the FE nodes at this green line is also restrained in order to avoid zero-energy modes. The discretisation contains 6,400 hexagonal trilinear FEs. 40 FEs are used in the axial direction, 40 in the circumferential direction and 4 in the radial direction.

The cross-sectional deformations at the tube's left end (blue) and right end (red) are presented in Fig. 13 together with the initial cross sections (green) for the different hyperelastic models. The force-displacement res- 


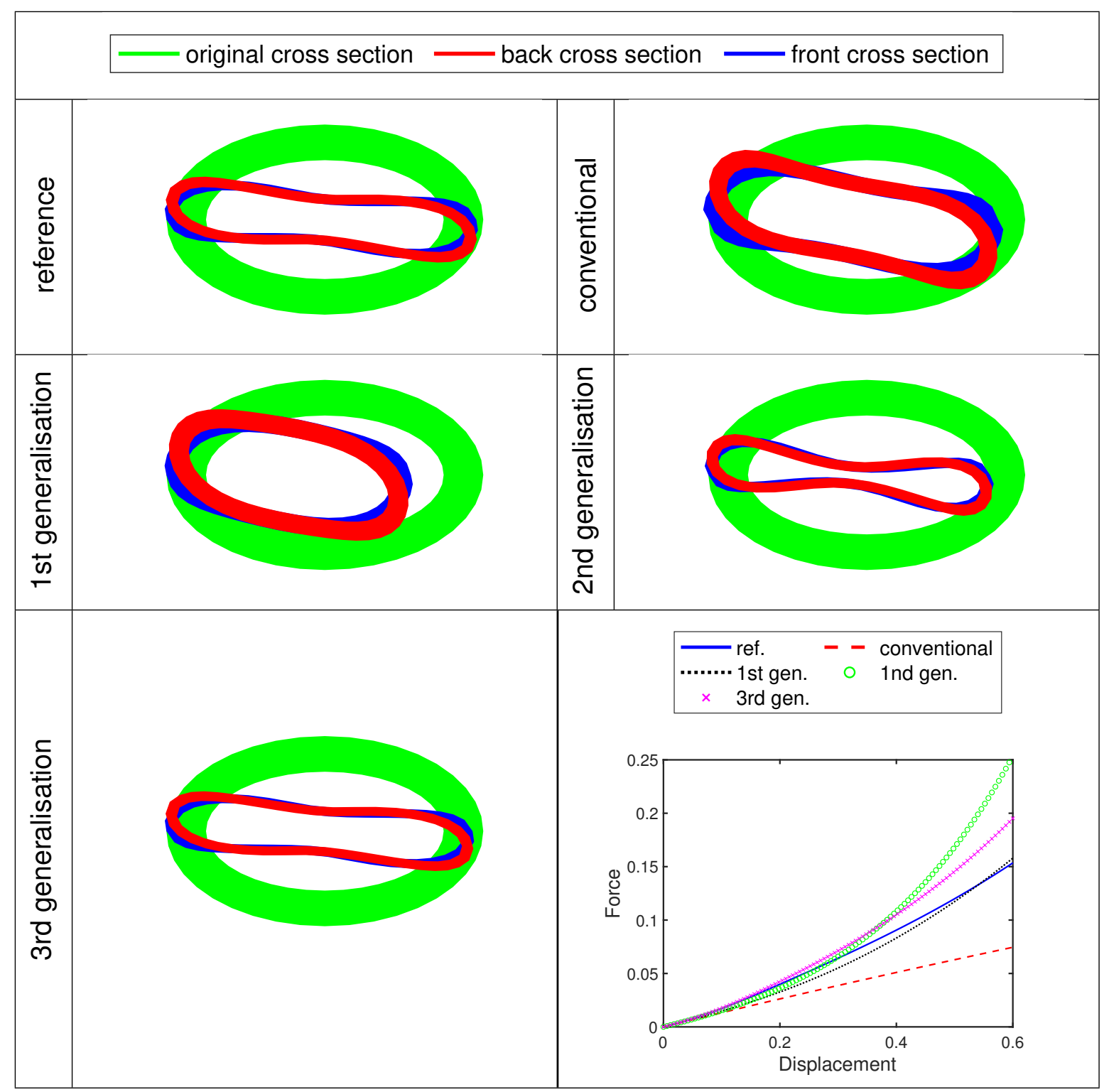

Figure 13: Propogation of unconventional material parameters for orthotropy: Deformations and force-displacement curves for the elliptical tube of Fig. 12. The deformations are not scaled. 
ponses are also presented, which are in accordance with the trends of the inverse model responses.

From the cross-sectional deformations in Fig. 13 we can learn several things. First, the cross sectional deformations of the reference case for which the third generalisation is used (top-left) is highly similar to those of the third generalisation with the identified parameters (bottom-left). This indicates that the shear response is not necessarily needed to identify the parameter values and hence, the compression and tensile data are sufficient, since a substantial amount of shear deformation is present in the tube, which is not considered in the identification.

Second, the cross sectional deformations predicted by the conventional hyperelasticity (top-right) are substantially different from all generalisations, indicating again that the generalisations are substantially different from the conventional hyperelasticity. Third, the cross sectional deformations predicted by the first generalisation (centre-left) are substantially different from those predicted by the second and third generalisation (top-left, centre-right and bottom-left), indicating that the first generalisation is substantially different from the other two (even though the identified inverse model responses are similar to that of the second generalisation). Fourth, the cross sectional deformations predicted by the second generalisation (centre-right) show most similarities with those of the third generalisation (top-left and bottom-left), as both the second and third generalisation gather two strain tensors in one, whereas the first generalisation gathers two strain energy densities in one. This again shows that the first generalisation is different from the second and third one.

\section{Conclusion}

Strain energy densities quadratically expressed in terms of the Lagrangian strain tensors of the Seth-Hill family can amongst others be used to describe compressible isotropic, transversely isotropic and orthotropic hyperelastic deformations. Thanks to its relatively accurate physical character however, the Hencky strain tensor is probably a frequently employed family member. A true experimental identification of the most suitable strain tensor of the Seth-Hill family is thus not often performed.

To offer the possibility to fit the material response in the non-linear regime, this contribution has proposed three generalisations for compressible hyperelasticity based on the Seth-Hill strain tensors. All generalisations 
enable the incorporation of several members of the Seth-Hill family in a single material model. All generalisations also reduce to the conventional hyperelasticity, if only one Seth-Hill strain tensor is incorporated. All generalisations furthermore recover the infinitesimal theory for small deformations and rotations, meaning that the identification of the material parameters for the nonlinear regime does not interfere with the identification of the standard material parameters (Young's moduli, Poisson's ratios and shear moduli). It must be noted that the generalisations are not polyconvex (see e.g. Schröder and Neff (2003); Balzani et al. (2006)).

Although the results have clearly shown that the three generalisation are truly different from each other, the second generalisation is perhaps the most attractive thanks to three reasons. First, the implementation efforts are truly minimal (for implementations that do not use the Green-Lagrange strain tensor), since only a few scalars need to be altered. Second, it expresses all employed conventional strain tensors in a single strain tensor, whereas a single strain tensor is not trivial to distinguish in the first generalisation. Third, the associated FE computations require substantially less time than for the first generalisation.

As the generalisations allow to identify material behaviours in the nonlinear regime, tensile and compression data are required, and true inverse models are needed. This study has however shown that by selecting appropriate specimen dimensions, it is sufficient to select a single material point without shear contributions as the inverse model for the tensile test and a single finite element as the the inverse model for the compression test. Hence, the computational costs of the required inverse modelling are not as demanding as one may expect at first sight.

\section{References}

Andrade F.X.C., Cesár de Sá J.M.A., Andrade Pires F.M., A ductile damage nonlocal model of integral-type at finite strains: formulation and numerical issues, International Journal of Damage Mechanics 20 (2011) 515-557.

Baaijens F.P.T., Trickey W.R., Laursen T.A., Guilak F., Large deformation finite element analysis of micropipette aspiration to determine the mechanical properties of the chondrocyte, Annals of Biomedical Engineering 33 (2005) 492-499. 
Balzani D., Neff P., Schröder J., Holzapfel G.A., A polyconvex framework for soft biological tissues. Adjustment to experimental data, International Journal of Solids and Structures 43 (2006) 6052-6070.

Beex L.A.A., Peerlings R.H.J., On the influence of delamination on laminated paperboard creasing and folding, Philosophical Transactions of the Royal Society A 370 (2012) 1912-1924.

Bernstein B., Hypo-elasticity and elasticity, Archive for Rational Mechanics and Analysis 6 (1960a) 89-104.

Bernstein B., Relations between hypoelasticity and elasticity, Transactions of the Society of Rheology 4 (1960b) 23-28.

Bui H.P., Tomar S., Courtecuisse H., Cotin S., Bordas S.P.A., Real-time error control for surgical simulation, IEEE Transactions on Biomedical Engineering 65 (2018) 596-607.

Carnial T.A., Fancello E.A., A transversely isotropic coupled hyperelastic model for the mechanical behavior of tendons, Journal of Biomechanics 54 (2017) 49-57.

Cotin S., Delingette H., Ayache N., Real-time elastic deformations of soft tissues for surgery simulation, IEEE Transactions on Visualization and Computer Graphics 5 (1999) 62-73.

Crespo J., Latorre M., Montáns F.J., WYPIWYG hyperelasticity for isotropic, compressible materials, Computational Mechanics 59 (2016) 7392.

Curnier A., Rakotomanana L., Generalized strain and stress measures: critical survey and new results, Engineering Transactions 39 (1991) 461-538.

Doyle T.C., Ericksen J.L., Nonlinear elasticity. Advances in Applied Mechanics 4 (1956) 53-115.

Geers M.G.D., Finite strain logarithmic hyperelasto-plasticity with softening: a strongly non-local implicit gradient framework, Computer Methods in Applied Mechanics and Engineering 193 (2004) 3377-3401. 
Håkansson P., Wallin M., Ristinmaa M., Comparison of isotropic hardening and kinematic hardening in thermoplasticity, Journal of Plasticity 21 (2005) 1435-1460.

Harrysson A., Ristinmaa M., Large strain elasto-plastic model of paper and corrugated board, International Journal of Solids and Structures 45 (2008) 3334-3352.

Hill R., On constitutive inequalities for simple materials-I, Journal of the Mechanics and Physics of Solids 16 (1968) 229-242.

Hill R., Aspects of invariance in solid mechanics, Advances in Applied Mechanics 18 (1978) 1-75.

Hrapko N., Van Dommelen J.A.W., Peters G.W., Wismans J.S., The mechanical behaviour of brain tissue: large strain response and constitutive modelling, Biorheology 43 (2006) 623-636.

Javani H.R., Peerlings R.H.J., Geers M.G.D., Consistent remeshing and transfer for a three dimensional enriched mixed formulation of plasticity and non-local damage, Computational Mechanics 53 (2014) 625-639.

Khan M.A., Mabrouki T., Vidal-Sallé E., Boisse P., Numerical and experimental analyses of woven composite reinforcement forming using a hypoelastic behaviour. Application to the double dome benchmark, Journal of Materials Processing Technology 210 (2010) 378-388.

Korobeynikov S.N., Objective symmetrically physical strain tensors, conjugate stress tensors, and Hill's linear isotropic hyperelastic material models, Journal of Elasticity 136 (2018) 159-187.

Kucheyev S.O., Stadermann M., Shin S.J., Satcher Jr. J.H., Gammon S.A., Letts S.A., Van Buuren T., Hamza A.V., Super-compressibility of ultralowdensity nanoporous silica, Advanced Materials 24 (2012) 776-780.

Latorre M., Montáns F.J., Stress and strain mapping tensors and general work-conjugacy in large strain continuum mechanics, Applied Mathematical Modelling 40 (2016) 3938-3950.

Latorre M., Montáns F.J., WYPiWYG hyperelasticity without inversion formula: Application to passive ventricular myocardium, Computers \& Structures 185 (2017) 47-58. 
Latorre M., Montáns F.J., Experimental data reduction for hyperelasticity, Computers \& Structures, In Press.

Loew P., Peters B., Beex L.A.A., Rate-dependent phase-field damage modeling of rubber and its experimental parameter identification, Journal of the Mechanics and Physics of Solids 127 (2019) 266-294.

Lovadina C., Stenberg R., Energy norm a posteriori error estimates for mixed finite element methods, Mathematics of Computation 75 (2006) 1659-1674.

Mahnken A., Shaban A., Finite elasto-viscoplastic modeling of polymers including asymmetric effects, Archive of Applied Mechanics 83 (2013) 53-71.

Miehe C., Lambrecht M., Algorithms for computation of stresses and elasticity moduli in terms of Seth-Hill's family of generalized strain tensors, Communications in Numerical Methods in Engineering 17 (2001) 337-353.

Miehe C., Apel N., Lambrecht M., Anisotropic additive plasticity in the logarithmic strain space: modular kinematic formulation and implementation based on incremental minimization principles for standard materials, Computer Methods in Applied Mechanics and Engineering 191 (2002) 53835425 .

Mihai L.A., Goriely A., Positive or negative Poynting effect? The role of adscititious inequalities in hyperelastic materials, Proceedings of the Royal Society A 467 (2011) 3633-3646.

Mihai L.A., Goriely A., How to characterize a nonlinear elastic material? A review on nonlinear constitutive parameters in isotropic finite elasticity, Proceedings of the Royal Society A 473 (2017) 20170607.

Nocedal J., Wright S.J., Numerical optimisation - Second Edition, Springer (2006) ISBN-10: 0-387-30303-0, ISBN-13: 978-0387-30303-1.

Ogden R.W., Large deformation isotropic elasticity- on the correlation of theory and experiment for incompressible rubber-like solids, Proceedings of the Royal Society A 328 (1972) 567-584.

Ogden R.W., Non-linear elastic deformations, Ellis Horwood Ltd. (1984) ISBN 0-85 312-273-3. 
Pokorný P, Šmejkal F., Kulmon P., Novák P., Novák J., Mikš A., Horák M., Jirásek M., Deformation of a prestressed liquid lens membrane, Applied Optics 56 (2017) 9368-9376.

Polak E., Ribiere G., Note sur la convergence de méthodes de directions conjugée, Revue Française d'Informatique et de Recherche Opérationnelle Série Rouge 3 (1969) 35-43.

Romero X., Latorre M., Montáns F.J., Determination of the WYPiWYG strain energy density of skin through finite element analysis of the experiments on circular specimens, Finite Elements in Analysis and Design 134 (2017) 1-15.

Rosa De E., Latorre M., Montáns F.J., Capturing anisotropic constitutive models with WYPiWYG hyperelasticity; and on consistency with the infinitesimal theory at all deformation levels, International Journal of Nonlinear Mechanics 196 (2017) 75-92.

Seth B.R., Generalized strain measure with applications to physical problems, IUTAM Symposium on second-order effects in elasticity, plasticity and fluid mechanics, Haifa (1962).

Schaedler T.A., Jacobsen A.J., Torrents A., Sorensen A.E., Lian J., Greer J.R., Valdevit L., Carter W.B., Ultralight metallic microlattices, Science 334 (2011) 962-964.

Schröder J., Gruttmann F., Löbleim J., A simple orthotropic finite elastoplasticity model based on generalized stress-strain measures, Computational Mechanics 30 (2002) 48-64.

Schröder J., Neff P., Invariant formulation of hyperelastic transverse isotropy based on polyconvex free energy functions, International Journal of Solids and Structures 40 (2003) 401-445.

Treloar L.R.G., The physics of rubber elasticity - second edition, Oxford University Press (1958).

Truesdell C., Hypo-elasticity, Journal of Rational Mechanics and Analysis 4 (1955) 83-133. 
Vogel F., Göktepe S., Steinmann P., Kuhl E., Modeling and simulation of viscous electro-active polymers, European Journal of Mechanics A.Solids 48 (2014) 112-128.

Wismans J.G.F., Govaert L.E., Van Dommelen J.A.W., X-Ray computed tomography-based modeling of polymeric foams: the effect of finite element model size on the large strain response, Journal of Polymer Science B 48 (2010) 1526-1534.

Xiao H. Bruhns O.T., Meyers A., Hypo-elasticity model based upon the logarithmic stress rate, Journal of Elasticity 47 (1997) 51-68.

Xiao H. Chen L.S., Hencky's elasticity model and linear stress-strain relations in isotropic finite hyperelasticity, Acta Mechanica 157 (2002) 51-60.

Zienkiewicz O.C., Taylor R.L., The finite element method, volume 1 - fifth edition, Butterworth-Heinemann (2000). 